Supporting Information for

\title{
Synthesis and Characterization of Tungsten Alkylidene and Alkylidyne Complexes Featuring a New Carbazole-Based Rigid Trianionic $\mathrm{ONO}^{3-}$ Pincer-Type Ligand
}

Ushnish Mandal, Sudarsan VenkatRamani, Ion Ghiviriga, Khalil A. Abboud, and Adam S. Veige*

University of Florida, Department of Chemistry, Center for Catalysis, P.O. Box 117200, Gainesville, FL, 32611.

Table of Contents

Experimental Section......................................................... S3

General Consideration...................................................... S3

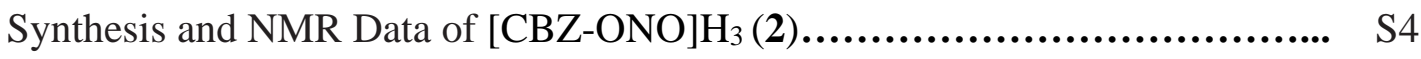

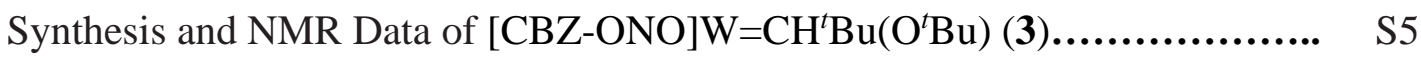

Synthesis and NMR Data of $\left\{\mathrm{CH}_{3} \mathrm{Ph}_{3} \mathrm{P}\right\}\left\{[\mathrm{CBZ}-\mathrm{ONO}] \mathrm{W} \equiv \mathrm{C}^{t} \mathrm{Bu}\left(\mathrm{O}^{t} \mathrm{Bu}\right)\right\}(\mathbf{4}) \ldots \ldots . \quad$ S6

Addition of MeOTf to 4 and generation of [CBZ-ONO]W $\equiv \mathrm{O}\left(\mathrm{CH}_{2}{ }^{t} \mathrm{Bu}\right)(\mathbf{6})$ in a $\quad \mathrm{S} 6$

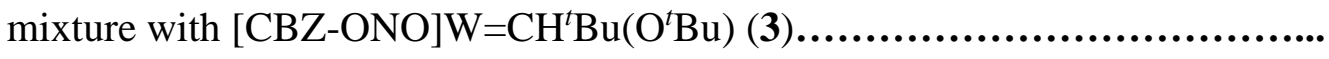

In situ synthesis of [CBZ-ONO]W $\equiv \mathrm{O}\left(\mathrm{CH}_{2}{ }^{t} \mathrm{Bu}\right)(\mathbf{6})$ and isobutylene expulsion.. $\quad \mathrm{S} 7$

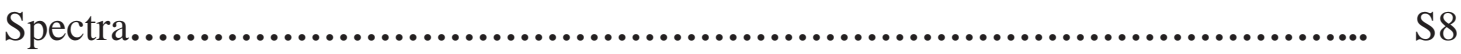

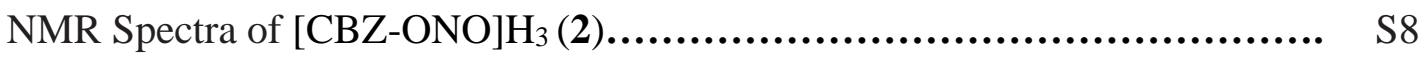

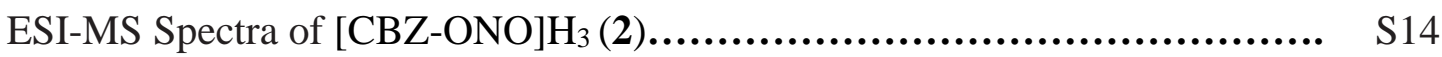

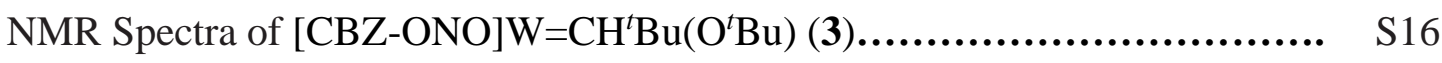

NMR Spectra of $\left\{\mathrm{CH}_{3} \mathrm{Ph}{ }_{3} \mathrm{P}\right\}\left\{[\mathrm{CBZ}-\mathrm{ONO}] \mathrm{W} \equiv \mathrm{C}^{t} \mathrm{Bu}\left(\mathrm{O}^{t} \mathrm{Bu}\right)\right\}(\mathbf{4}) \ldots \ldots \ldots \ldots \ldots \ldots \ldots . . . . . . . \quad \mathrm{S} 23$

NMR Spectra of [CBZ-ONO]W $\equiv \mathrm{O}\left(\mathrm{CH}_{2}{ }^{t} \mathrm{Bu}\right)(\mathbf{6})$ in a mixture with $\quad$ S29

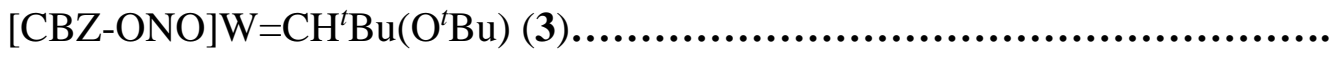


NMR Spectra for the in situ generation of [CBZ-ONO]W $\equiv \mathrm{O}\left(\mathrm{CH}_{2}{ }^{t} \mathrm{Bu}\right)(\mathbf{6})$ and

Isobutylene expulsion

UV-Vis Study and Spectra................................................. S38

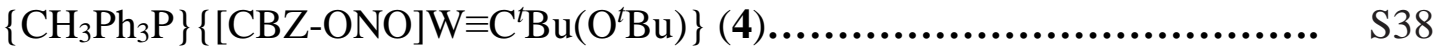

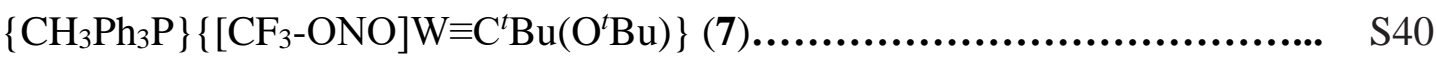

X-ray Crystal Structure Data.................................................. S42

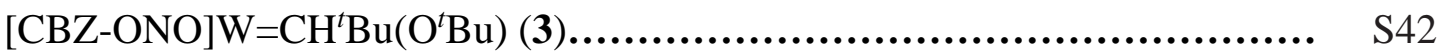

Computational Methods....................................................... S45

General Consideration for computed model complexes $\mathbf{3}^{\prime}, \mathbf{4}^{\prime}$ and $\mathbf{7}^{\prime} \ldots \ldots \ldots \ldots . . . . \quad$ S45

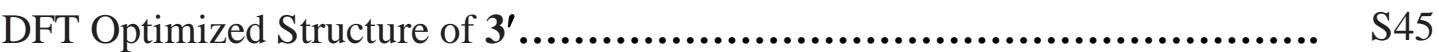

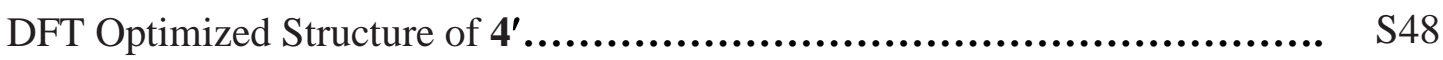

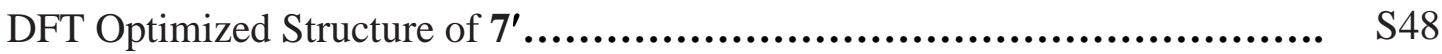

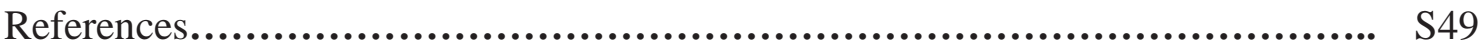




\section{$\underline{\text { Experimental Section }}$}

\section{General Consideration}

Unless specified otherwise, all manipulations were performed under an inert atmosphere using standard Schlenk or glove box techniques. Glassware was oven-dried before use. Pentane, hexane, toluene, diethyl ether $\left(\mathrm{Et}_{2} \mathrm{O}\right)$, tetrahydrofuran $(\mathrm{THF})$, benzene $\left(\mathrm{C}_{6} \mathrm{H}_{6}\right)$ were dried using a GlassContours drying column and stored over $3 \AA$ molecular sieves. Benzene- $d_{6}$ (Cambridge Isotopes) was dried over sodium-benzophenone ketyl, distilled, and stored over $3 \AA$ molecular sieves. Chlorofom- $d_{1}$ (Cambridge Isotopes) was dried over $\mathrm{CaH}_{2}$, vacuum transferred and stored over $3 \AA$ molecular sieves. 1,8-dibromo-3,6-dimethyl-9H-carbazole, ${ }^{1} \quad\left({ }^{t} \mathrm{BuO}\right)_{3} \mathrm{~W} \equiv \mathrm{C}^{t} \mathrm{Bu}^{2}$, $\mathrm{PPh}_{3} \mathrm{CH}_{2}{ }^{3,4}$ and complex $\left\{\mathrm{CH}_{3} \mathrm{Ph}_{3} \mathrm{P}\right\}\left\{\left[\mathrm{CF}_{3}-\mathrm{ONO}\right] \mathrm{W} \equiv \mathrm{C}^{t} \mathrm{Bu}\left(\mathrm{O}^{t} \mathrm{Bu}\right)\right\}(7)^{5}$ were prepared according to published procedures. All other reagents were purchased from commercial vendors and used without further purification. NMR spectra were obtained on a Varian Mercury spectrometer operating at $300 \mathrm{MHz}$ for ${ }^{1} \mathrm{H},{ }^{19} \mathrm{~F}$ and ${ }^{31} \mathrm{P} .{ }^{13} \mathrm{C}$ and $2 \mathrm{D}$ NMR spectra were acquired from either Varian Inova spectrometer operating at $500 \mathrm{MHz}$ or Bruker $600 \mathrm{MHz}$ spectrometer. ${ }^{15} \mathrm{~N}$ NMR spectra were recorded in Bruker $600 \mathrm{MHz}$ spectrometer. The chemical shifts are reported in $\delta$ (ppm), referenced to the lock signal on the TMS scale for ${ }^{1} \mathrm{H}$ and ${ }^{13} \mathrm{C} \mathrm{NMR} \mathrm{spectra,} \mathrm{CFCl}_{3}$ for ${ }^{19} \mathrm{~F}$ NMR spectra, neat $\mathrm{H}_{3} \mathrm{PO}_{4}$ scale for ${ }^{31} \mathrm{P}$ NMR spectra, and ammonia for ${ }^{15} \mathrm{~N}$. ESI-MS analysis was performed at the Mass Spectrometry Research and Education Center, UF Chemistry Department. Elemental analyses were performed at the CENTC Elemental Analysis facility at University of Rochester, NY.

Caution: hexafluoroacetone is a highly volatile and toxic gas and must be used in a well-ventilated fume hood. 


\section{Synthesis of [CBZ-ONO]H 3 (2).}

Inside a nitrogen-filled glovebox, a solution of $n$-butyllithium $(11.0 \mathrm{~mL}, 1.60 \mathrm{M}$ in hexanes, $17.6 \mathrm{mmol}$ ) was added dropwise to a Schlenk flask containing a solution of 1,8-dibromo-3,6dimethyl-9H-carbazole $(2.00 \mathrm{~g}, 5.66 \mathrm{mmol})$ in $\mathrm{Et}_{2} \mathrm{O}(100 \mathrm{~mL})$ at $-35{ }^{\circ} \mathrm{C}$. The yellow colored reaction mixture was stirred for $2 \mathrm{~h}$ while warming to room temperature. The reaction flask was fitted with a dry ice condenser before exiting the box. The reaction flask was connected to a Schlenk line through the port on the dry ice condenser. The reaction solution was cooled to -78 ${ }^{\circ} \mathrm{C}$, and dry ice and acetone were added to the condenser. Inside a fume hood Hexafluoroacetone (2.86 mL, $3.77 \mathrm{~g}, 22.7 \mathrm{mmol}$ ) was first condensed into a graduated glass pressure flask by condensing at $-78{ }^{\circ} \mathrm{C}$. The pressure flask containing hexafluoroacetone was then connected to the reaction flask via a side arm. The pressure flask was allowed to slowly warm to room temperature causing the hexafluoroacetone to condense into the reaction-flask producing a brown colored reaction mixture. The reaction mixture was allowed to warm to room temperature while the dry ice/acetone condenser was kept filled for $3 \mathrm{~h}$ (the hexafluoroacetone will condense on the cold finger and drip back into the solution). After $3 \mathrm{~h}$, the dry ice/acetone was no longer replenished and was allowed to warm, and the reaction mixture stirred overnight. To the resulting red solution was added $\mathrm{HCl}_{(\mathrm{aq})}(19 \mathrm{~mL}, 1.00 \mathrm{M})$ and transferred to a separatory funnel. The organic layer was collected and stored over anhydrous $\mathrm{MgSO}_{4}$. The aqueous layer was again extracted with $\mathrm{Et}_{2} \mathrm{O}$ (30 $\mathrm{mL}$ ) and the extracts stored over anhydrous $\mathrm{MgSO}_{4}$. The combined extracts were then filtered and reduced under vacuum to a thick oil. The oil was then dried for $4 \mathrm{~h}$ on a Schlenk line at $60{ }^{\circ} \mathrm{C}$ under dynamic vacuum. Pentane $(50 \mathrm{~mL})$ was added to the oil to produce a yellow solution and a black solid. The mixture was filtered, and the filtrate in pentane was reduced under vacuum, and triturated with acetone. The residual acetone was removed under vacuum and the resulting oil was 
precipitated by addition of a minimal amount of pentane to yield a white (faint yellowish) powder which was isolated by filtration ( $1.16 \mathrm{~g}, 39 \%$ yield). Note: if a precipitate does not form upon the second addition of pentane, additional trituration with acetone can be done. ${ }^{1} \mathrm{H}$ NMR (300 MHz, $\left.\mathrm{C}_{6} \mathrm{D}_{6}, 25^{\circ} \mathrm{C}\right): \delta 9.96(\mathrm{~s}, 1 \mathrm{H}, \mathrm{N}-H), 7.66(\mathrm{~s}, 2 \mathrm{H}, \mathrm{Ar}-H), 7.58(\mathrm{~s}, 2 \mathrm{H}, \mathrm{Ar}-H), 2.82(\mathrm{~s}, 2 \mathrm{H}, \mathrm{O}-H), 2.25$ (s, $\left.6 \mathrm{H}, \mathrm{CH}_{3}\right)$ ppm. ${ }^{19} \mathrm{~F}$ NMR $\left(282 \mathrm{MHz}, \mathrm{C}_{6} \mathrm{D}_{6}, 25{ }^{\circ} \mathrm{C}\right): \delta \quad-74.8\left(\mathrm{~s}, 12 \mathrm{~F},-\mathrm{C} F_{3}\right) \mathrm{ppm} .{ }^{13} \mathrm{C}\left\{{ }^{1} \mathrm{H}\right\} \mathrm{NMR}$ $\left(125 \mathrm{MHz}, \mathrm{C}_{6} \mathrm{D}_{6}, 25^{\circ} \mathrm{C}\right): \delta 136.8$ (s, $\left.\operatorname{Ar}-C\right), 128.6$ (s, Ar-C), 126.0 (s, Ar-C), 124.8 (s, $\left.\operatorname{Ar}-C\right), 123.0$ $(\mathrm{s}, \operatorname{Ar}-C), 111.6(\mathrm{~s}, \operatorname{Ar}-C), 21.3(\mathrm{~s}, \mathrm{Ar}-\mathrm{CH} 3)$. ESI-MS: $[2+\mathrm{H}]^{+}=528.0851 ;[2+\mathrm{H}]^{+}$theoretical $=$ $528.0827(4.5 \mathrm{ppm})$.

\section{Synthesis of $[\mathrm{CBZ}-\mathrm{ONO}] \mathrm{W}=\mathrm{CH}^{t} \mathrm{Bu}\left(\mathrm{O}^{t} \mathrm{Bu}\right)(3)$.}

Inside the glovebox a benzene- $d_{6}$ solution $(1.00 \mathrm{~mL})$ containing $2\left(0.023 \mathrm{~g}, 4.36 \times 10^{-5} \mathrm{~mol}\right)$ was added drop-wise to a benzene- $d_{6}(1.00 \mathrm{~mL})$ solution of $\left({ }^{t} \mathrm{BuO}\right)_{3} \mathrm{~W} \equiv \mathrm{C}^{t} \mathrm{Bu}\left(0.021 \mathrm{~g}, 4.36 \times 10^{-5}\right.$ mol) in a sealable NMR tube. The NMR tube was then taken outside of the glovebox and was heated for $6 \mathrm{~h}$ at $50^{\circ} \mathrm{C}$. After heating, the NMR tube was brought inside the glovebox and all the volatiles were evaporated under dynamic vacuum for $4 \mathrm{~h}$. The solid was then triturated with pentane $(3 \mathrm{x})$. The brownish-red solid was then dissolved in pentane and filtered to remove any solid impurities. The filtrate was collected and dried under dynamic vacuum to obtain $\mathbf{3}$ as a brownred powder ( $0.027 \mathrm{~g}, 73 \%$ yield). Analytically pure $\mathbf{3}$ was obtained by recrystallizing it in cold ($35^{\circ} \mathrm{C}$ ) pentane (54\% yield). ${ }^{1} \mathrm{H}$ NMR $\left(300 \mathrm{MHz}, \mathrm{C}_{6} \mathrm{D}_{6}, 25^{\circ} \mathrm{C}\right): \delta 7.85(\mathrm{~s}, 2 \mathrm{H}, \mathrm{Ar}-H), 7.57(\mathrm{~s}, 2 \mathrm{H}$, Ar- $H), 6.58\left(\mathrm{~s}, 1 \mathrm{H}, \mathrm{W}=\mathrm{C} H^{t} \mathrm{Bu},{ }^{2} J\left({ }^{1} \mathrm{H},{ }^{183} \mathrm{w}\right)=8.80 \mathrm{~Hz}\right), 2.24\left(\mathrm{~s}, 6 \mathrm{H}, \mathrm{Ar}-\mathrm{CH}_{3}\right), 1.26(\mathrm{~s}, 9 \mathrm{H}$, $\left.\mathrm{OC}\left(\mathrm{CH}_{3}\right)_{3}\right), 1.12\left(\mathrm{~s}, 9 \mathrm{H}, \mathrm{WCH}\left(\mathrm{CH}_{3}\right)_{3}\right) \mathrm{ppm} .{ }^{19} \mathrm{~F}$ NMR $\left(282 \mathrm{MHz}, \mathrm{C}_{6} \mathrm{D}_{6}, 25{ }^{\circ} \mathrm{C}\right): \delta \quad-71.55(\mathrm{q}, 6 \mathrm{~F}$, $\left.-\mathrm{C} F_{3},{ }^{4} J_{\mathrm{FF}}=8.46 \mathrm{~Hz}\right),-74.93\left(\mathrm{q}, 6 \mathrm{~F},-\mathrm{C} F_{3},{ }^{4} J_{\mathrm{FF}}=8.46 \mathrm{~Hz}\right) \mathrm{ppm} .{ }^{13} \mathrm{C}\left\{{ }^{1} \mathrm{H}\right\} \mathrm{NMR}\left(125 \mathrm{MHz}, \mathrm{C}_{6} \mathrm{D}_{6}\right.$, $25^{\circ} \mathrm{C}$ ): $\delta 264.1\left(\mathrm{~s}, \mathrm{~W}=C \mathrm{H}^{t} \mathrm{Bu}\right), 140.9$ (s, $\left.\mathrm{Ar}-C\right), 132.4$ (s, $\left.\mathrm{Ar}-C\right), 127.4$ (s, $\mathrm{Ar}-C$ ), 125.4 (s, Ar-C), $122.4(\mathrm{~s}, \operatorname{Ar}-C), 118.2(\mathrm{~s}, \operatorname{Ar}-\mathrm{C}), 92.8\left(\mathrm{~s}, \mathrm{OC}\left(\mathrm{CH}_{3}\right)_{3}\right), 42.1\left(\mathrm{~s}, \mathrm{WCHC}\left(\mathrm{CH}_{3}\right)_{3}\right), 35.7(\mathrm{~s}$, 
WCHC $\left.\left(\mathrm{CH}_{3}\right)_{3}\right), 29.5\left(\mathrm{~s}, \mathrm{OC}\left(\mathrm{CH}_{3}\right)_{3}\right), 21.2\left(\mathrm{~s}, \mathrm{Ar}-\mathrm{CH}_{3}\right)$. Anal. Calcd. for $\mathrm{C}_{29} \mathrm{H}_{29} \mathrm{~F}_{12} \mathrm{NO}_{3} \mathrm{~W}(851.38$ g/mol): C, 40.91\%; H, 3.43\%; N, 1.65\%. Found: C, 40.25\%; H, 3.43\%; N, 1.75\%.

\section{Synthesis of $\left\{\mathrm{CH}_{3} \mathrm{Ph}{ }_{3} \mathrm{P}\right\}\left\{[\mathrm{CBZ}-\mathrm{ONO}] \mathrm{W} \equiv \mathrm{C}^{t} \mathrm{Bu}\left(\mathrm{O}^{t} \mathrm{Bu}\right)\right\}(4)$.}

A pentane solution $(5.00 \mathrm{~mL})$ of $\mathrm{Ph}_{3} \mathrm{PCH}_{2}\left(0.020 \mathrm{~g}, 7.16 \times 10^{-5} \mathrm{~mol}\right)$ was added dropwise to a stirring pentane solution of $\mathbf{3}\left(0.061 \mathrm{~g}, 7.16 \times 10^{-5} \mathrm{~mol}\right)$ resulting in the precipitation of a yellow powder. The mixture was stirred for $3 \mathrm{~h}$ and the solid was collected by filtration and dried under vacuum for $2 \mathrm{~h}$ to yield 4 as a yellow solid ( $0.064 \mathrm{~g}, 80 \%$ yield). ${ }^{1} \mathrm{H}$ NMR (300 MHz, $\mathrm{C}_{6} \mathrm{D}_{6}, 25$ $\left.{ }^{\circ} \mathrm{C}\right): \delta 7.97(\mathrm{~s}, 2 \mathrm{H}, \mathrm{Ar}-H), 7.91(\mathrm{~s}, 2 \mathrm{H}, \mathrm{Ar}-H), 6.98-6.67(b r \mathrm{~s}, 15 \mathrm{H}, \mathrm{Ar}-H), 2.48\left(\mathrm{~s}, 6 \mathrm{H}, \mathrm{Ar}_{-} \mathrm{CH}_{3}\right)$, $1.92\left(\mathrm{~d}, 3 \mathrm{H}, \mathrm{CH}_{3} \mathrm{PPh}_{3},{ }^{2} J_{\mathrm{HP}}=13.2 \mathrm{~Hz}\right), 1.84\left(\mathrm{~s}, 9 \mathrm{H}, \mathrm{OC}\left(\mathrm{CH}_{3}\right)_{3}\right), 0.91\left(\mathrm{~s}, 9 \mathrm{H}, \mathrm{WCH}\left(\mathrm{CH}_{3}\right)_{3}\right) \mathrm{ppm}$. ${ }^{19} \mathrm{~F} \mathrm{NMR}\left(282 \mathrm{MHz}, \mathrm{C}_{6} \mathrm{D}_{6}, 25^{\circ} \mathrm{C}\right): \delta-70.62\left(\mathrm{q}, 6 \mathrm{~F},-\mathrm{CF}_{3},{ }^{4} \mathrm{JFF}_{\mathrm{FF}}=8.46 \mathrm{~Hz}\right),-75.47$ (q, 6F, $-\mathrm{CF}_{3},{ }^{4} J_{\mathrm{FF}}$ $=8.46 \mathrm{~Hz}) \mathrm{ppm} .{ }^{13} \mathrm{C}\left\{{ }^{1} \mathrm{H}\right\} \mathrm{NMR}\left(125 \mathrm{MHz}, \mathrm{C}_{6} \mathrm{D}_{6}, 25{ }^{\circ} \mathrm{C}\right): \delta 288.9\left(\mathrm{~s}, \mathrm{~W} \equiv C^{t} \mathrm{Bu}\right), 145.5(\mathrm{~s}, \mathrm{Ar}-\mathrm{C})$, $134.7(\mathrm{~s}, \operatorname{Ar}-C), 132.7(\mathrm{~s}, \operatorname{Ar}-C), 130.1(\mathrm{~s}, \operatorname{Ar}-C), 128.6(\mathrm{~s}, \operatorname{Ar}-C), 126.8$ (s, $\operatorname{Ar}-C), 124.9$ (s, $\operatorname{Ar}-C)$ 124.5 (s, Ar-C), 121.2 (s, $\operatorname{Ar}-C), 119.2$ (s, $\operatorname{Ar}-C), 119.1$ (s, Ar-C), 118.5 (s, $\operatorname{Ar}-C), 78.2$ (s, $\left.\mathrm{OC}\left(\mathrm{CH}_{3}\right)_{3}\right), 50.1\left(\mathrm{~s}, \mathrm{WCC}\left(\mathrm{CH}_{3}\right)_{3}\right), 33.8\left(\mathrm{~s}, \mathrm{WCC}\left(\mathrm{CH}_{3}\right)_{3}, 30.2\left(\mathrm{~s}, \mathrm{OC}\left(\mathrm{CH}_{3}\right)_{3}\right), 21.9\left(\mathrm{~s}, \mathrm{Ar}-\mathrm{CH}_{3}\right), 8.0\right.$ $\left(\mathrm{d}, \mathrm{H}_{3} \mathrm{CPPh}_{3},{ }^{1} \mathrm{~J}_{\mathrm{PC}}=235 \mathrm{~Hz}\right) .{ }^{31} \mathrm{P}\left\{{ }^{1} \mathrm{H}\right\} \mathrm{NMR}\left(121 \mathrm{MHz}, \mathrm{C}_{6} \mathrm{D}_{6}, 25^{\circ} \mathrm{C}\right): \delta 20.38$ (s) ppm. Anal. Calcd. for $\mathrm{C}_{48} \mathrm{H}_{46} \mathrm{~F}_{12} \mathrm{NO}_{3} \mathrm{PW}(1127.69 \mathrm{~g} / \mathrm{mol}): \mathrm{C}, 51.12 \% ; \mathrm{H}, 4.11 \%$; N, 1.24\%. Found: C, 50.66\%; H, $3.80 \% ; \mathrm{N}, 1.08 \%$.

\section{Addition of MeOTf to 4 and generation of $[\mathrm{CBZ}-\mathrm{ONO}] \mathrm{W} \equiv \mathrm{O}\left(\mathrm{CH}_{2}{ }^{t} \mathrm{Bu}\right)(6)$.}

A 4:1 $\mathrm{Et}_{2} \mathrm{O}$ and THF solution $(1.00 \mathrm{~mL})$ of $4\left(0.028 \mathrm{~g}, 2.48 \times 10^{-5} \mathrm{~mol}\right)$ was taken in a vial and cooled to $-35^{\circ} \mathrm{C}$. MeOTf $\left(0.003 \mathrm{~mL}, 2.60 \times 10^{-5} \mathrm{~mol}\right)$ after passing through basic alumina, was also dissolved in 4:1 $\mathrm{Et}_{2} \mathrm{O}$ and THF solution $(0.100 \mathrm{~mL})$ and cooled to $-35^{\circ} \mathrm{C}$. The cold MeOTf solution was then added to the stirring cold solution of 4 . The solution was allowed to warm to room 
temperature and stirred for $6 \mathrm{~h}$ after which it was filtered to remove white phosphonium salts. All volatiles were then removed in vacuo and the residue was triturated with pentane (3x). It was then dissolved in $\mathrm{Et}_{2} \mathrm{O}$, cooled, and filtered again to remove residual phosphonium salts. All the volatiles were stripped, and cold pentane was added to the residue. After filtration, the pentane solution was stripped to yield a yellowish powder, a mixture of $\mathbf{3}$ and $\mathbf{6}$ with other impurities [NMR conversion: $55 \%$ (6) and 45\% (3)]. ${ }^{1} \mathrm{H}$ NMR (300 MHz, $\left.\mathrm{C}_{6} \mathrm{D}_{6}, 25^{\circ} \mathrm{C}\right): \delta 7.84$ (s, $2 \mathrm{H}, \mathrm{Ar}-H$ of $\mathbf{3}$ ), $7.57\left(\mathrm{~s}, 2 \mathrm{H}, \mathrm{Ar}-H\right.$ of 3), $7.55(\mathrm{~s}, 2 \mathrm{H}, \mathrm{Ar}-H$ of $\mathbf{6}), 7.51(\mathrm{~s}, 2 \mathrm{H}, \mathrm{Ar}-H$ of 6$), 6.58\left(\mathrm{~s}, \sim 1 \mathrm{H}, \mathrm{W}=\mathrm{C} H^{t} \mathrm{Bu}\right.$ of 3), 3.09 (s, 2H, W-CH $H_{2}$ of 6), 2.24 (s, 6H, Ar-CH $\mathrm{CH}_{3}$ of 3), 2.19 (s, 6H, $\mathrm{Ar}_{-} \mathrm{CH}_{3}$ of $\left.\mathbf{6}\right), 1.43$ (s, 9H, W- $\mathrm{CH}_{2} \mathrm{C}\left(\mathrm{CH}_{3}\right)_{3}$ of $\left.\mathbf{6}\right), 1.25$ (s, 7H, $\mathrm{OC}\left(\mathrm{CH}_{3}\right)_{3}$ of $\left.\mathbf{3}\right), 1.12$ (s, 7H, WCH$\left(\mathrm{CH}_{3}\right)_{3}$ of $\left.\mathbf{3}\right)$ ppm. ${ }^{19} \mathrm{~F}$ NMR $\left(282 \mathrm{MHz}, \mathrm{C}_{6} \mathrm{D}_{6}, 25^{\circ} \mathrm{C}\right): \delta-71.55\left(\mathrm{q}, 4 \mathrm{~F},-\mathrm{CF}_{3}\right.$ of $\left.\mathbf{3},{ }^{4} J_{\mathrm{FF}}=8.46 \mathrm{~Hz}\right),-74.93\left(\mathrm{q}, 4 \mathrm{~F},-\mathrm{CF}_{3}\right.$ of $3,{ }^{4} J_{\mathrm{FF}}$ $=8.46 \mathrm{~Hz}),-73.48\left(\mathrm{q}, 6 \mathrm{~F},-\mathrm{C} F_{3}\right.$ of $\left.\mathbf{6},{ }^{4} J_{\mathrm{FF}}=8.46 \mathrm{~Hz}\right), \delta-74.33\left(\mathrm{q}, 6 \mathrm{~F},-\mathrm{C} F_{3}\right.$ of $\left.\mathbf{6},{ }^{4} J_{\mathrm{FF}}=8.46 \mathrm{~Hz}\right)$ ppm.

\section{In situ synthesis of $[\mathrm{CBZ}-\mathrm{ONO}] \mathrm{W} \equiv \mathrm{O}\left(\mathrm{CH}_{2}{ }^{t} \mathrm{Bu}\right)(6)$ and isobutylene expulsion.}

Inside the glovebox a benzene- $d_{6}$ solution $(0.200 \mathrm{~mL})$ containing MeOTf $\left(0.005 \mathrm{~mL}, 4.39 \times 10^{-5}\right.$ mol, after passing through basic alumina) was added drop-wise to a benzene- $d_{6}(0.600 \mathrm{~mL})$ solution of $\mathbf{3}\left(0.034 \mathrm{~g}, 4.00 \times 10^{-5} \mathrm{~mol}\right)$ in a sealable NMR tube. The NMR tube was then taken outside of the glovebox and was allowed to heat at $60{ }^{\circ} \mathrm{C}$ for $6 \mathrm{~h}$. Expulsion of isobutylene and generation of complex 6 were confirmed via ${ }^{1} \mathrm{H}$ NMR and ${ }^{19} \mathrm{~F}$ NMR spectroscopy after $6 \mathrm{~h}$ of heating. In the ${ }^{1} \mathrm{H}$ NMR spectrum a singlet for the methyl protons on the pincer ligand of $\mathbf{6}$ appears at $2.19 \mathrm{ppm}$, the ${ }^{t} \mathrm{Bu}$ protons $\mathrm{W}-\mathrm{CH}_{2} \mathrm{C}\left(\mathrm{CH}_{3}\right)_{3}$ resonate at $1.43 \mathrm{ppm}$, and the methylene protons of $\mathrm{W}-\mathrm{CH}_{2}$ appear at $3.08 \mathrm{ppm}$. Aromatic protons of $\mathbf{6}$ resonate at $7.56 \mathrm{ppm}$ and $7.51 \mathrm{ppm}$ Methyl protons of isobutylene resonate at $1.60 \mathrm{ppm}$ and the olefinic protons resonate at $4.75 \mathrm{ppm}$ 
confirming the expulsion of isobutylene. ${ }^{6}$ Also, the ${ }^{19} \mathrm{~F}$ NMR spectrum contains two quartets at $73.48\left({ }^{4} J_{\mathrm{FF}}=8.46 \mathrm{~Hz}\right) \mathrm{ppm}$ and $-74.33\left({ }^{4} J_{\mathrm{FF}}=8.46 \mathrm{~Hz}\right)$ ppm for complex $\boldsymbol{6}$.

\section{$\underline{\text { Spectra }}$}

NMR Characterization of [CBZ-ONO]H3 (2).

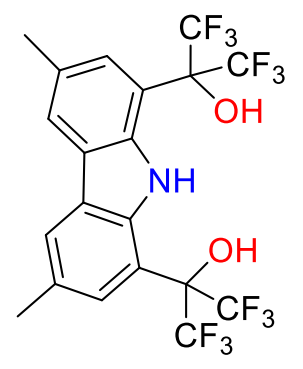

2

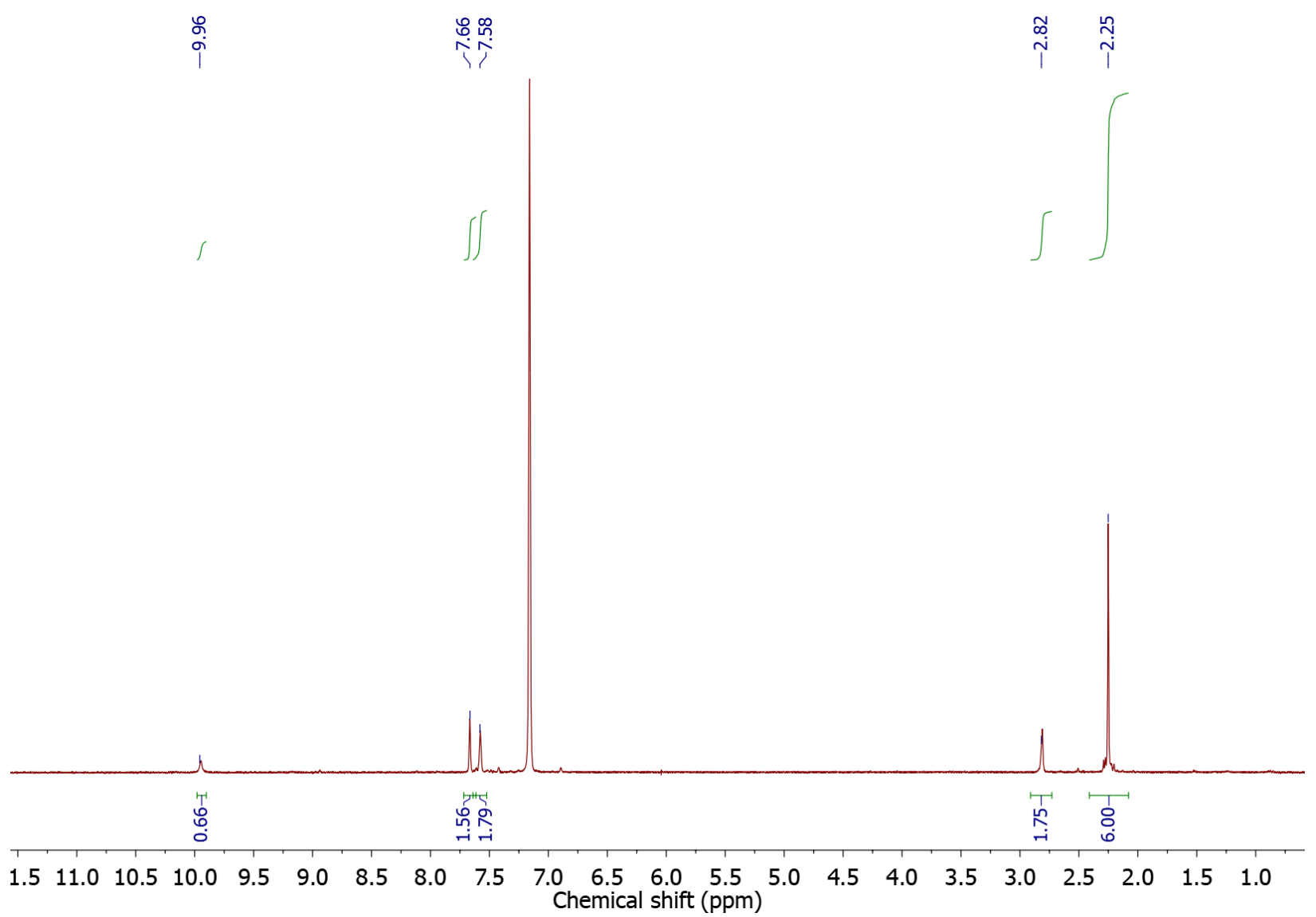

Figure S1. ${ }^{1} \mathrm{H}$ NMR spectrum of $2\left(\mathrm{C}_{6} \mathrm{D}_{6}, 300 \mathrm{MHz}, 25^{\circ} \mathrm{C}\right)$. 


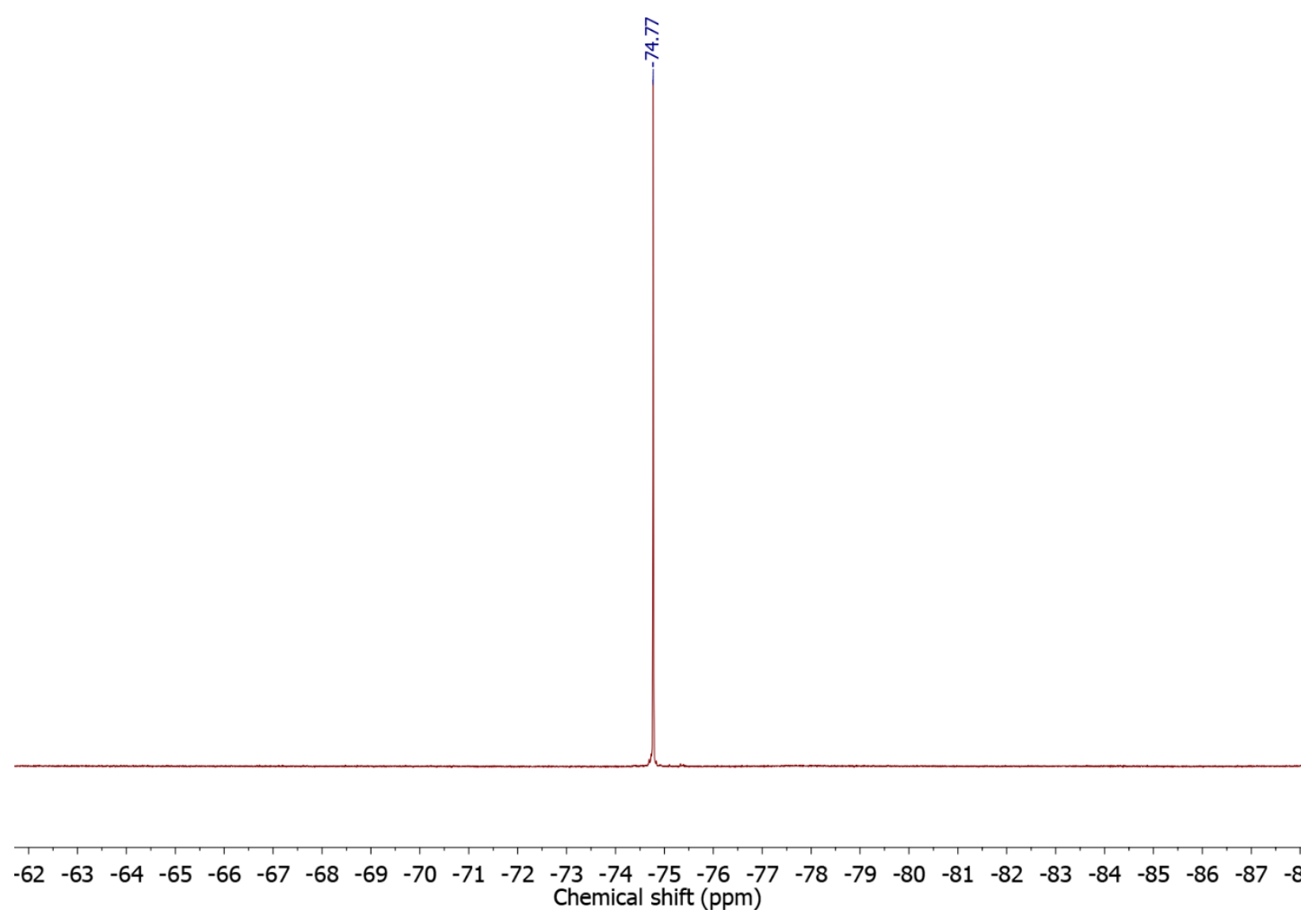

Figure S2. ${ }^{19} \mathrm{~F}$ NMR spectrum of $2\left(\mathrm{C}_{6} \mathrm{D}_{6}, 282 \mathrm{MHz}, 25{ }^{\circ} \mathrm{C}\right)$. 


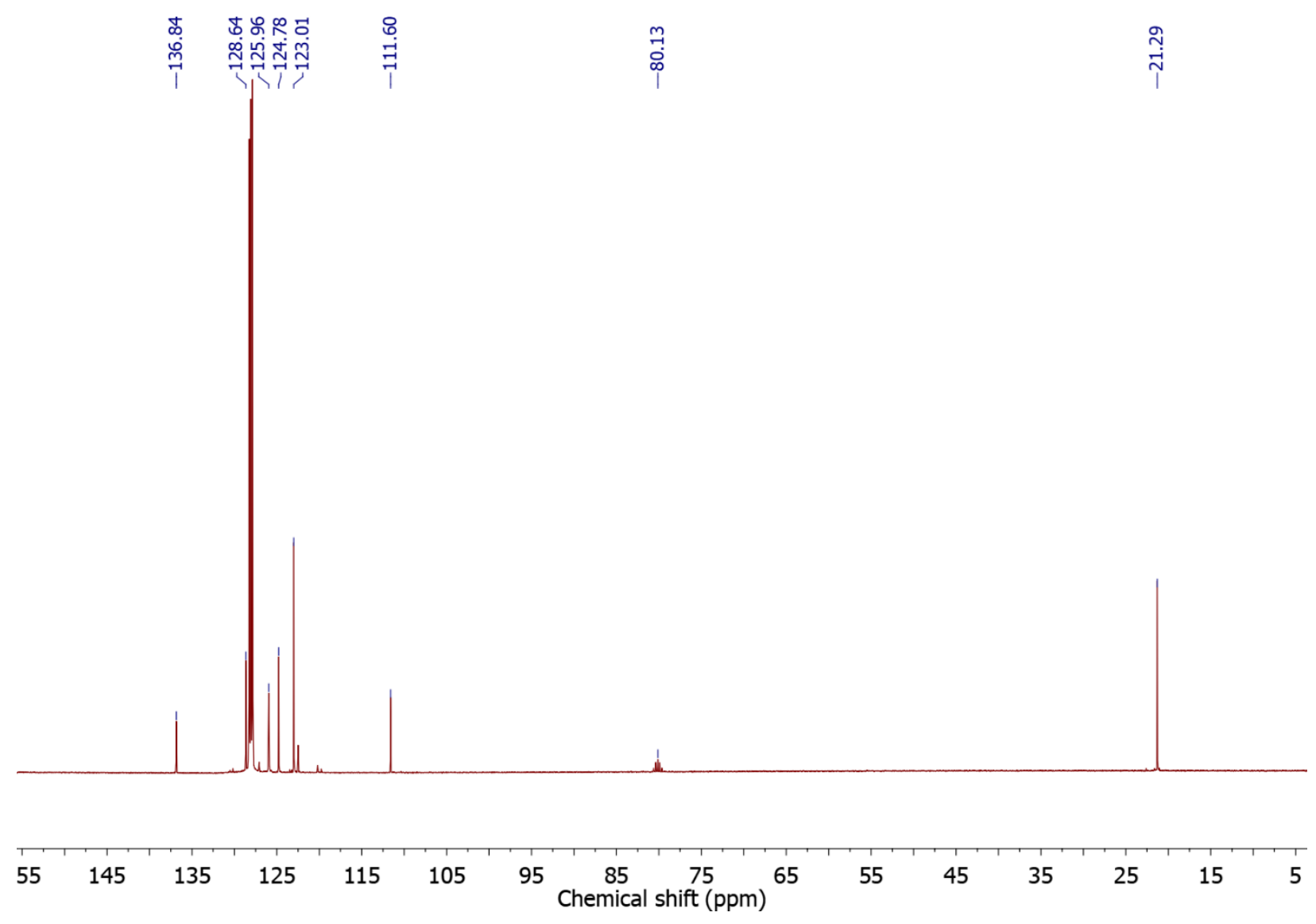

Figure S3. ${ }^{13} \mathrm{C}\left\{{ }^{1} \mathrm{H}\right\}$ NMR spectrum of $2\left(\mathrm{C}_{6} \mathrm{D}_{6}, 125 \mathrm{MHz}, 25{ }^{\circ} \mathrm{C}\right)$. 


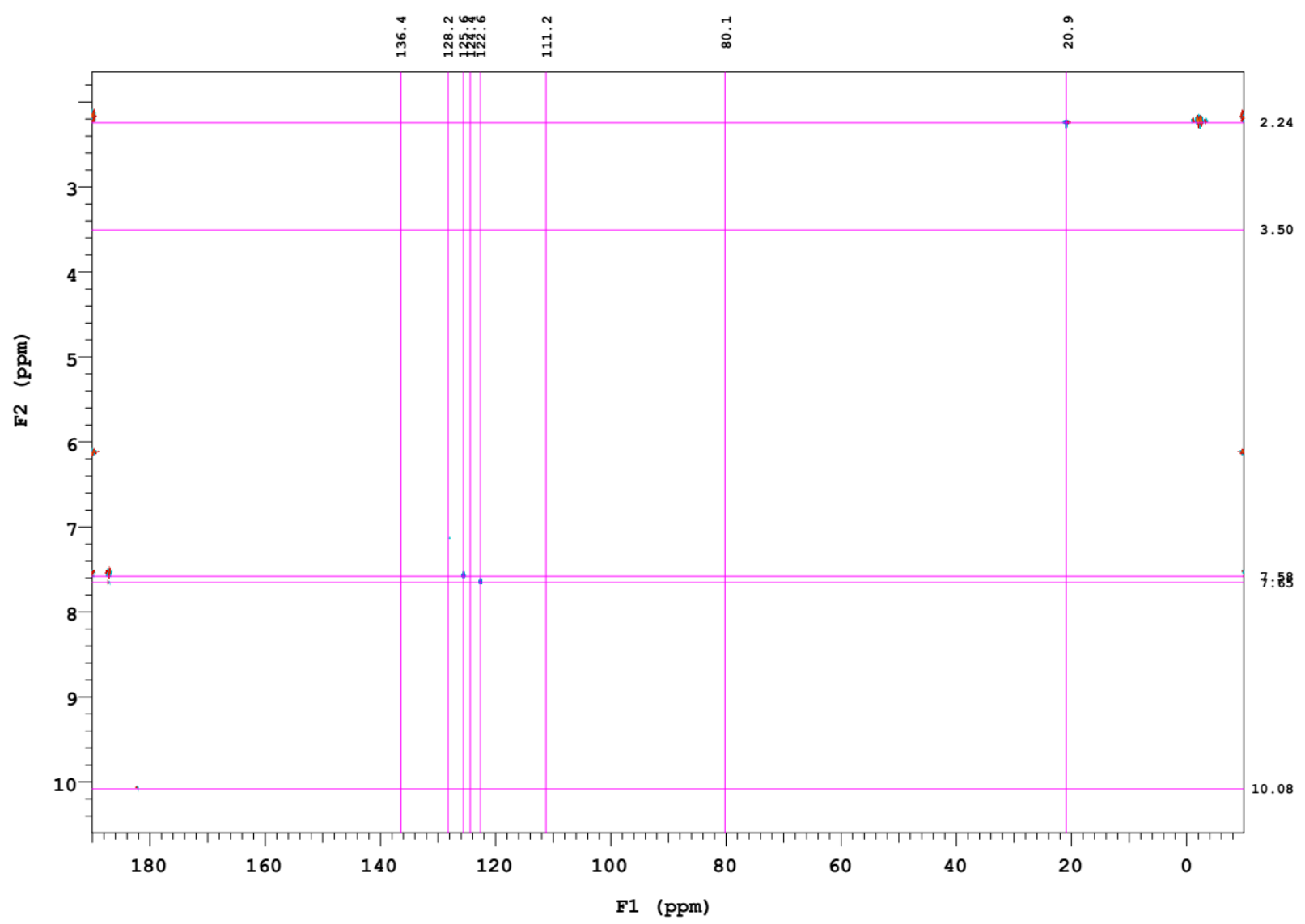

Figure S4. ${ }^{1} \mathrm{H}-{ }^{13} \mathrm{C}$ gHSQC spectrum of $2\left(\mathrm{C}_{6} \mathrm{D}_{6}, 500 \mathrm{MHz}, 25{ }^{\circ} \mathrm{C}\right)$. 


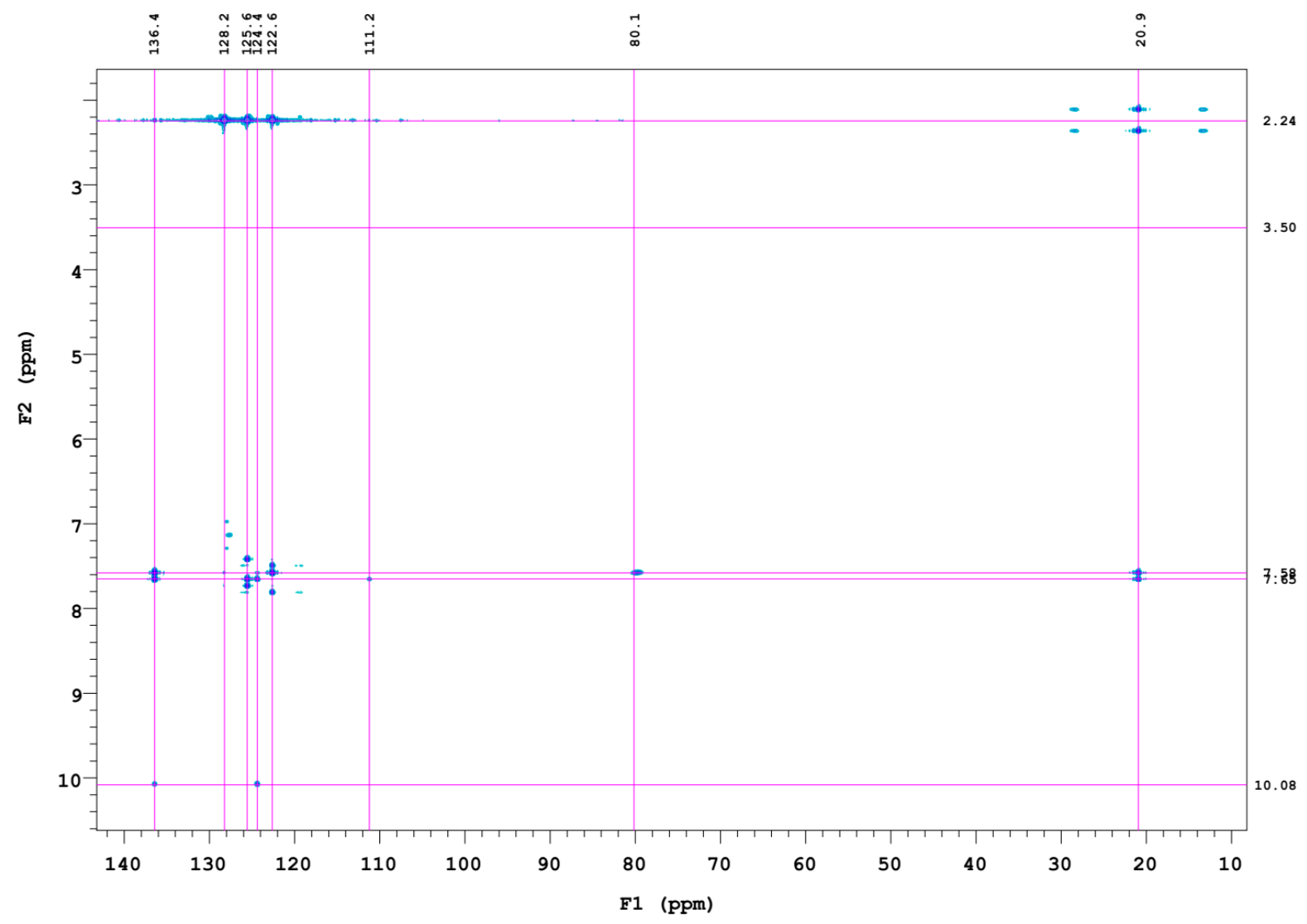

Figure S5. ${ }^{1} \mathrm{H}-{ }^{13} \mathrm{C}$ gHMBC spectrum of $2\left(\mathrm{C}_{6} \mathrm{D}_{6}, 500 \mathrm{MHz}, 25{ }^{\circ} \mathrm{C}\right)$. 


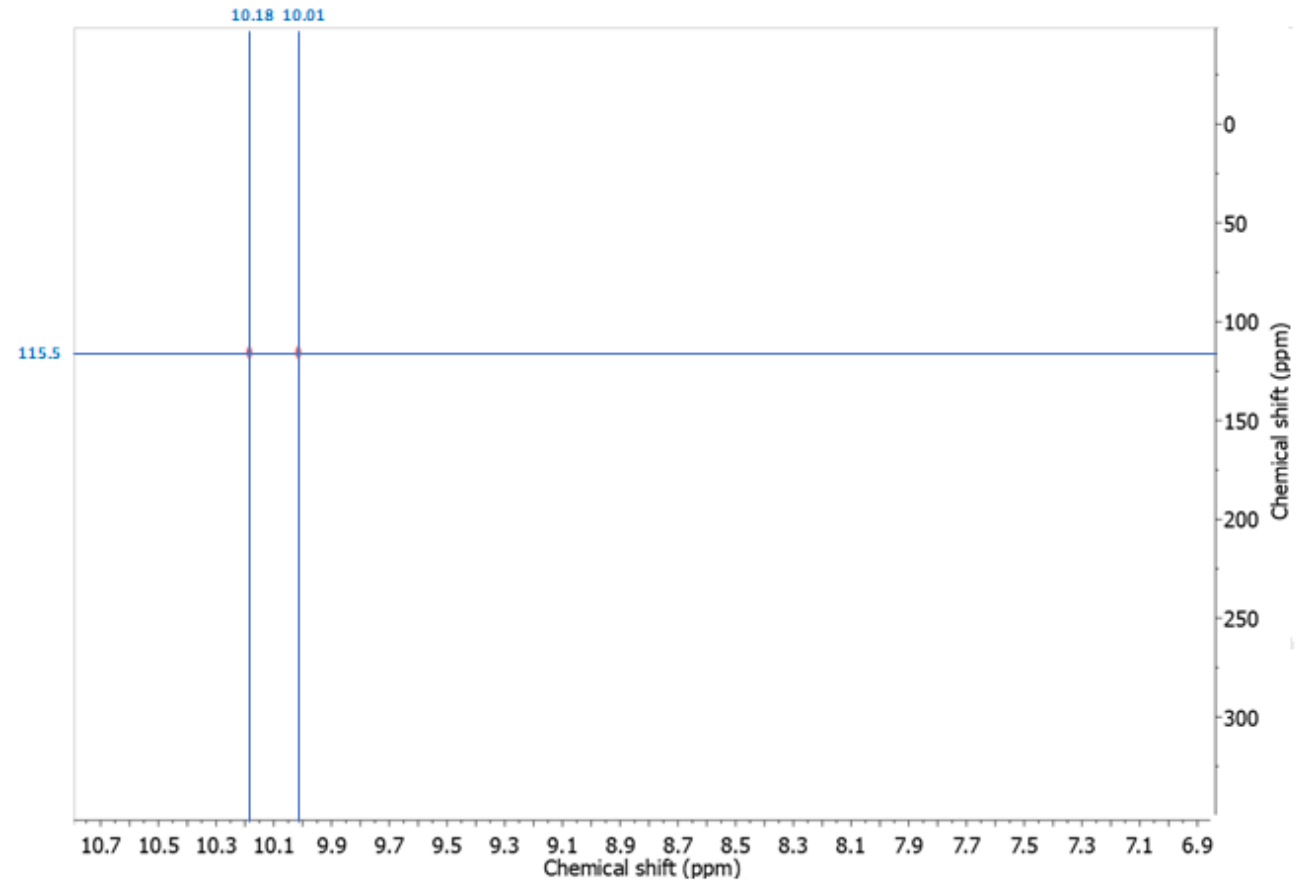

Figure S6. ${ }^{1} \mathrm{H}-{ }^{15} \mathrm{~N}$ gHMBC spectrum of $2\left(\mathrm{C}_{6} \mathrm{D}_{6}, 600 \mathrm{MHz}, 25{ }^{\circ} \mathrm{C}\right)$. 


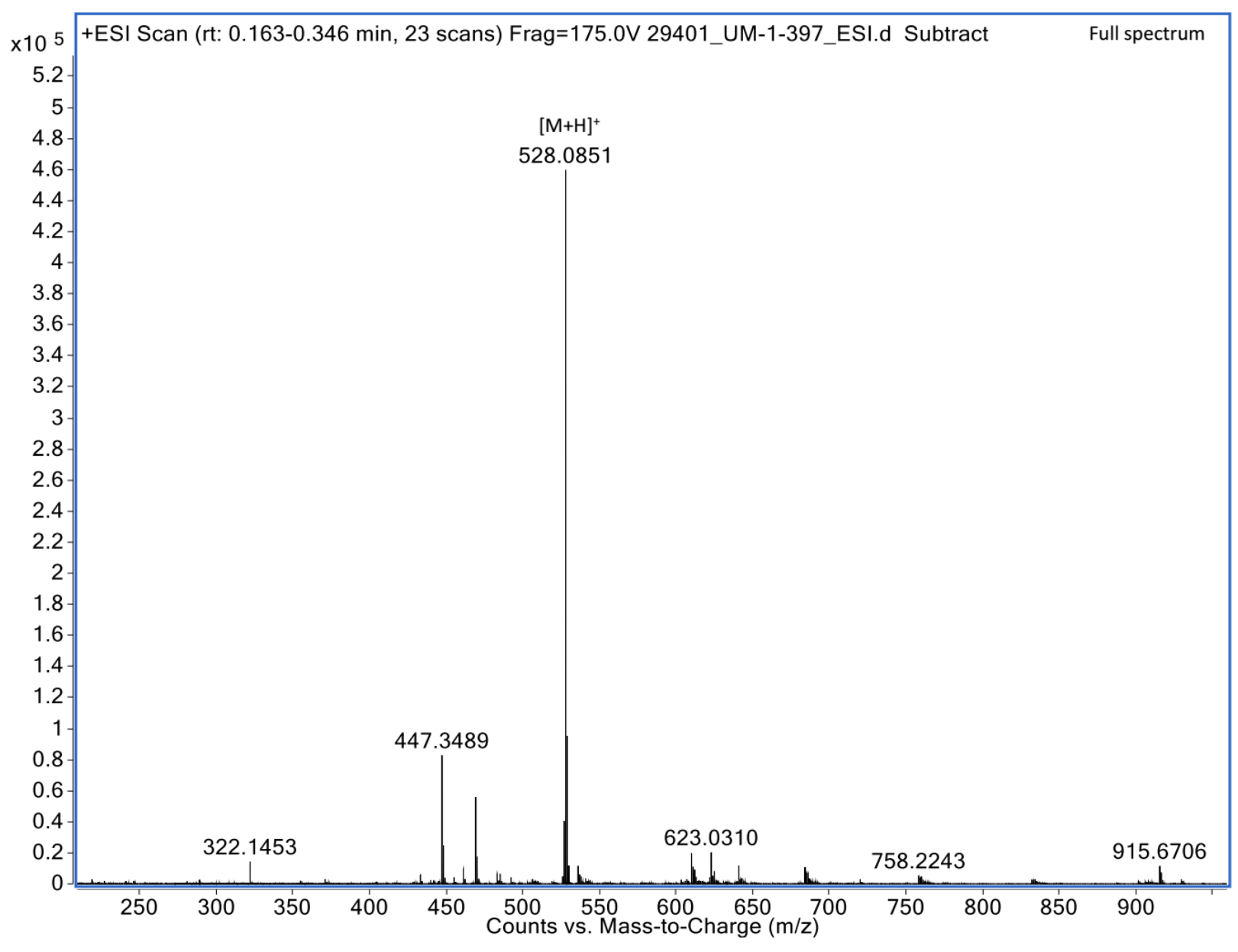

Figure S7. ESI-MS spectrum of 2 in methanol. $\left([2+\mathrm{H}]^{+}=528.0851 ;[2+\mathrm{H}]^{+}\right.$theoretical $=528.0827$ (4.5 ppm)). 


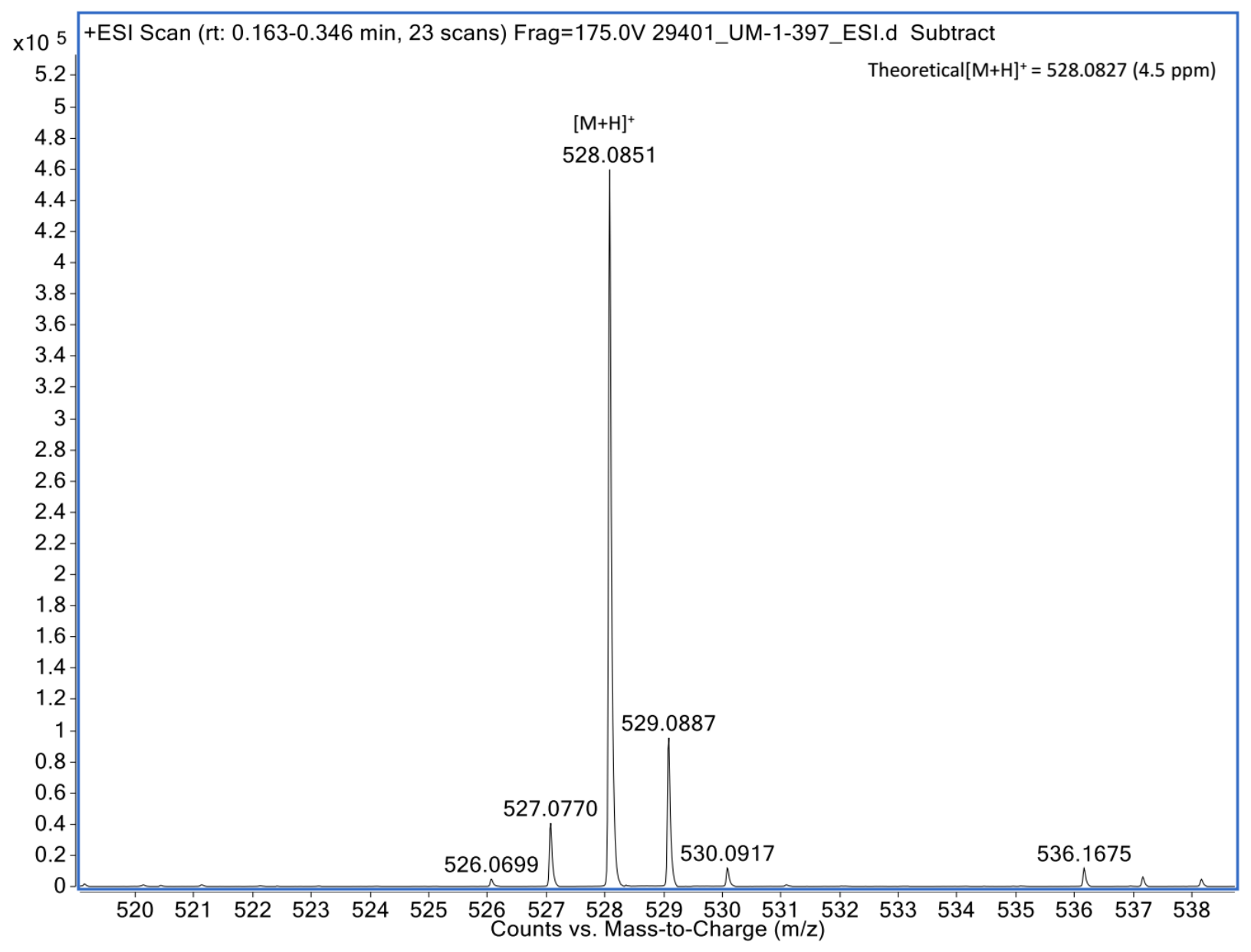

Figure S8. ESI-MS spectrum of 2 in methanol, expanded. $\left([2+\mathrm{H}]^{+}=528.0851 ;[2+\mathrm{H}]^{+}\right.$theoretical $=528.0827(4.5 \mathrm{ppm}))$. 
NMR Characterization of $[\mathrm{CBZ}-\mathrm{ONO}] \mathrm{W}=\mathrm{CH}^{t} \mathrm{Bu}\left(\mathrm{O}^{t} \mathrm{Bu}\right)(3)$.

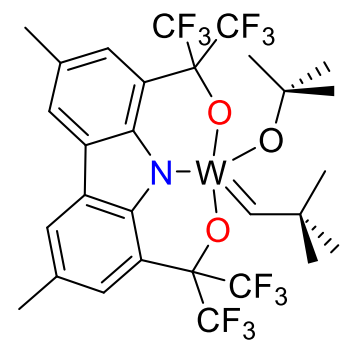

3

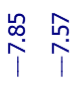

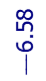
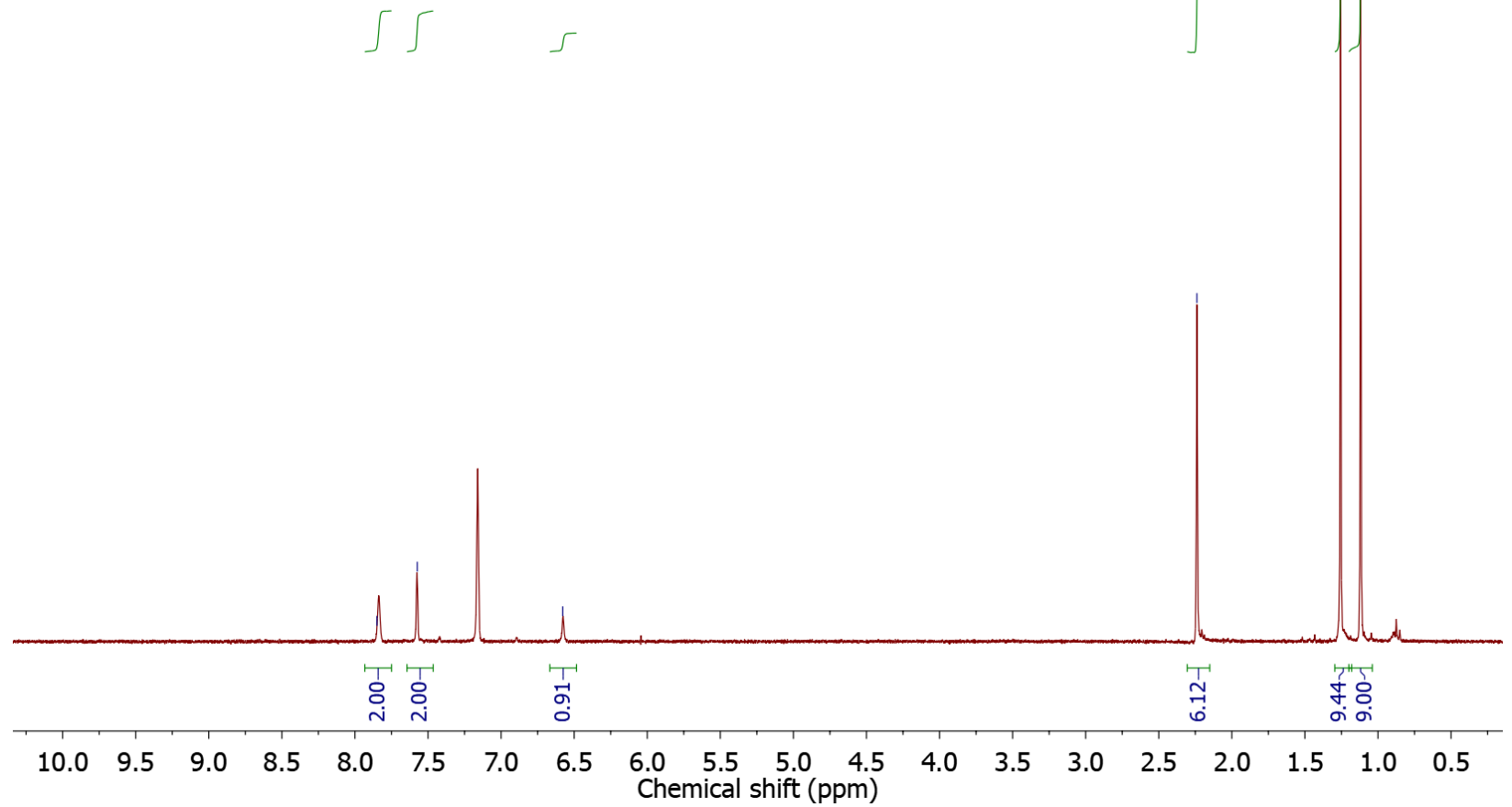

Figure S9. ${ }^{1} \mathrm{H}$ NMR spectrum of $\mathbf{3}\left(\mathrm{C}_{6} \mathrm{D}_{6}, 300 \mathrm{MHz}, 25{ }^{\circ} \mathrm{C}\right)$ 


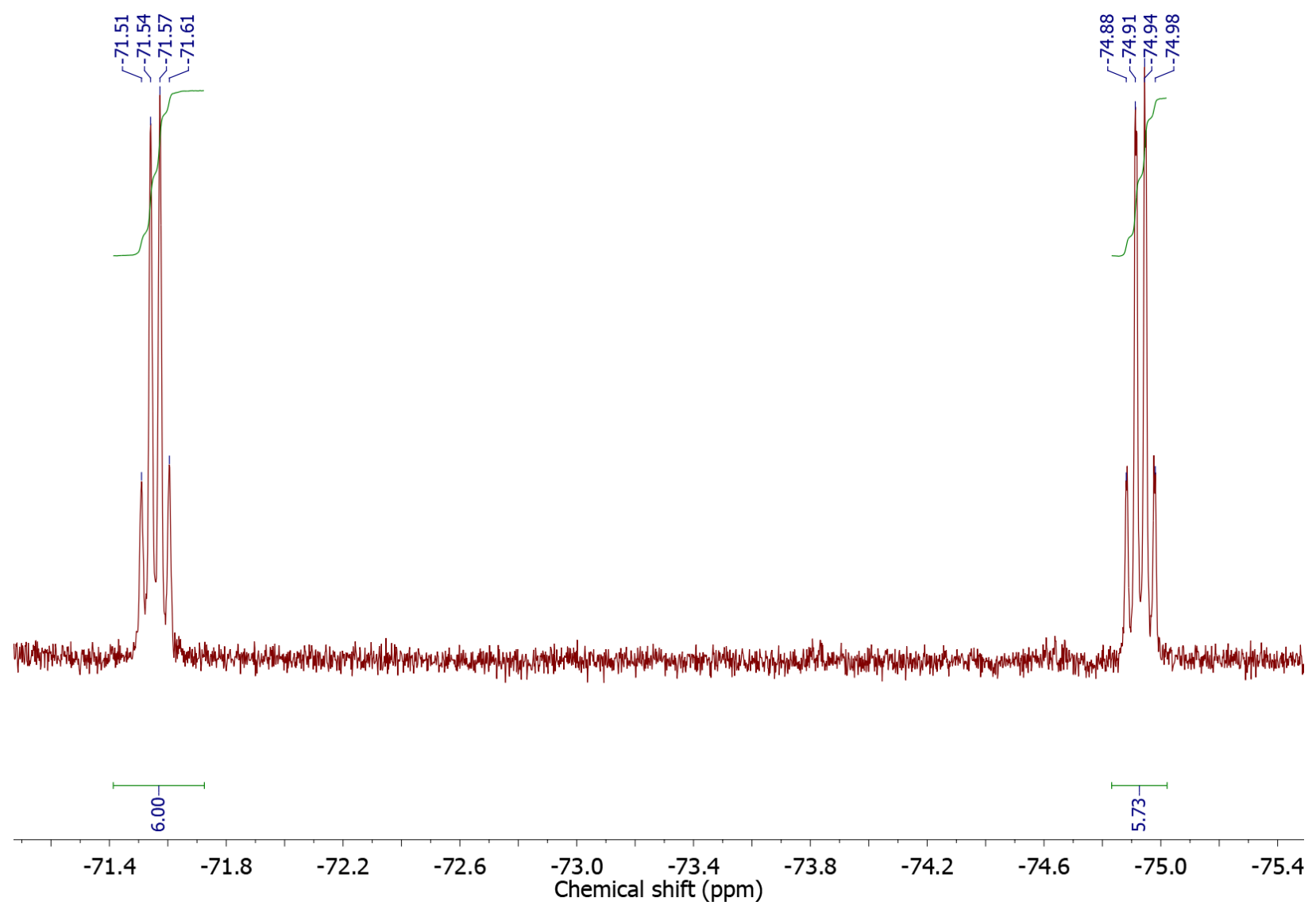

Figure S10. ${ }^{19} \mathrm{~F}$ NMR spectrum of $\mathbf{3}\left(\mathrm{C}_{6} \mathrm{D}_{6}, 282 \mathrm{MHz}, 25^{\circ} \mathrm{C}\right)$. 


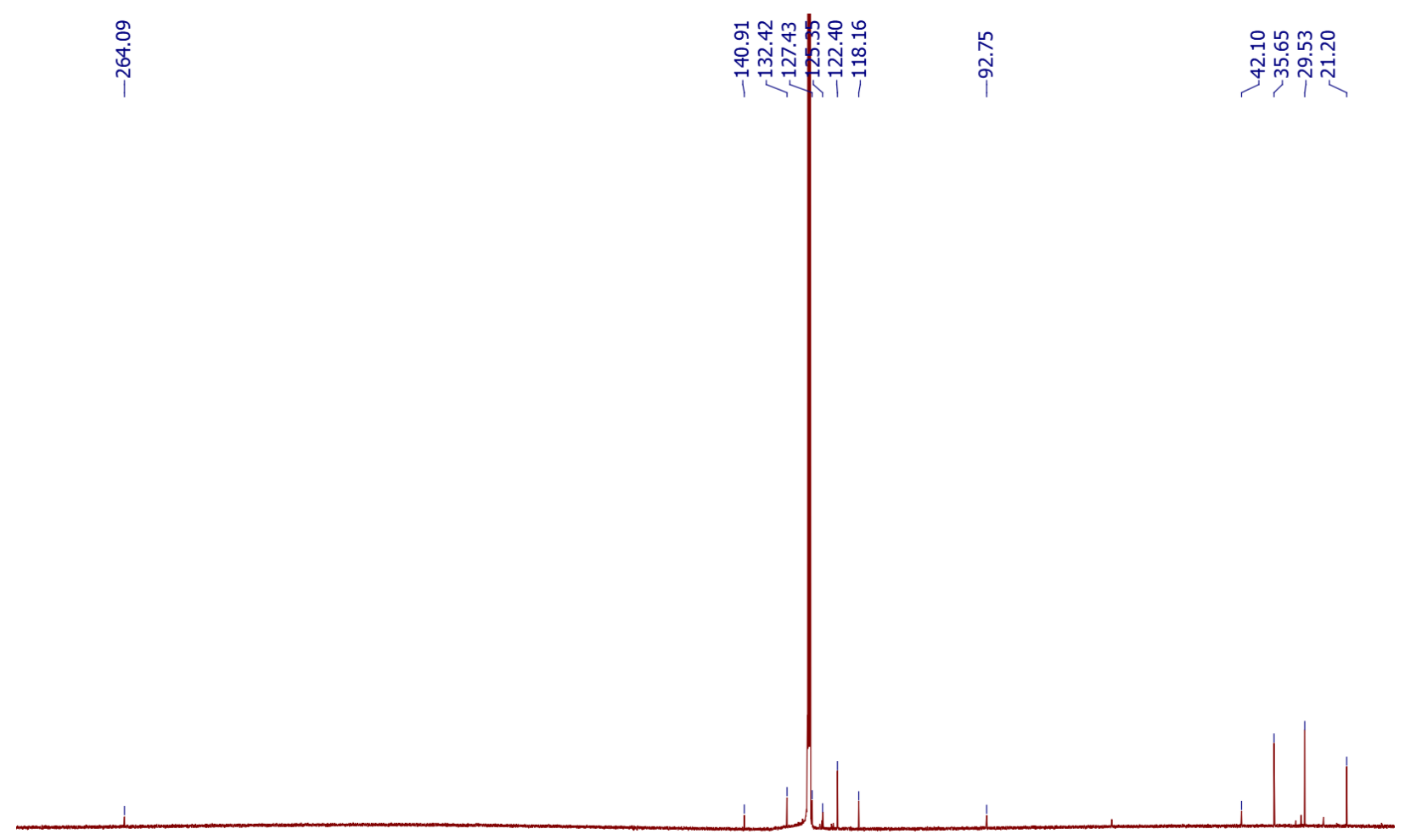

$280270260250240230220210200190180170160150140130120110100 \quad 90 \quad 80 \quad 70 \quad 60 \quad 50 \quad 40 \quad 30 \quad 20$ Chemical shift (ppm)

Figure S11. ${ }^{13} \mathrm{C}\left\{{ }^{1} \mathrm{H}\right\}$ NMR spectrum of $\mathbf{3}\left(\mathrm{C}_{6} \mathrm{D}_{6}, 125 \mathrm{MHz}, 25{ }^{\circ} \mathrm{C}\right)$. 


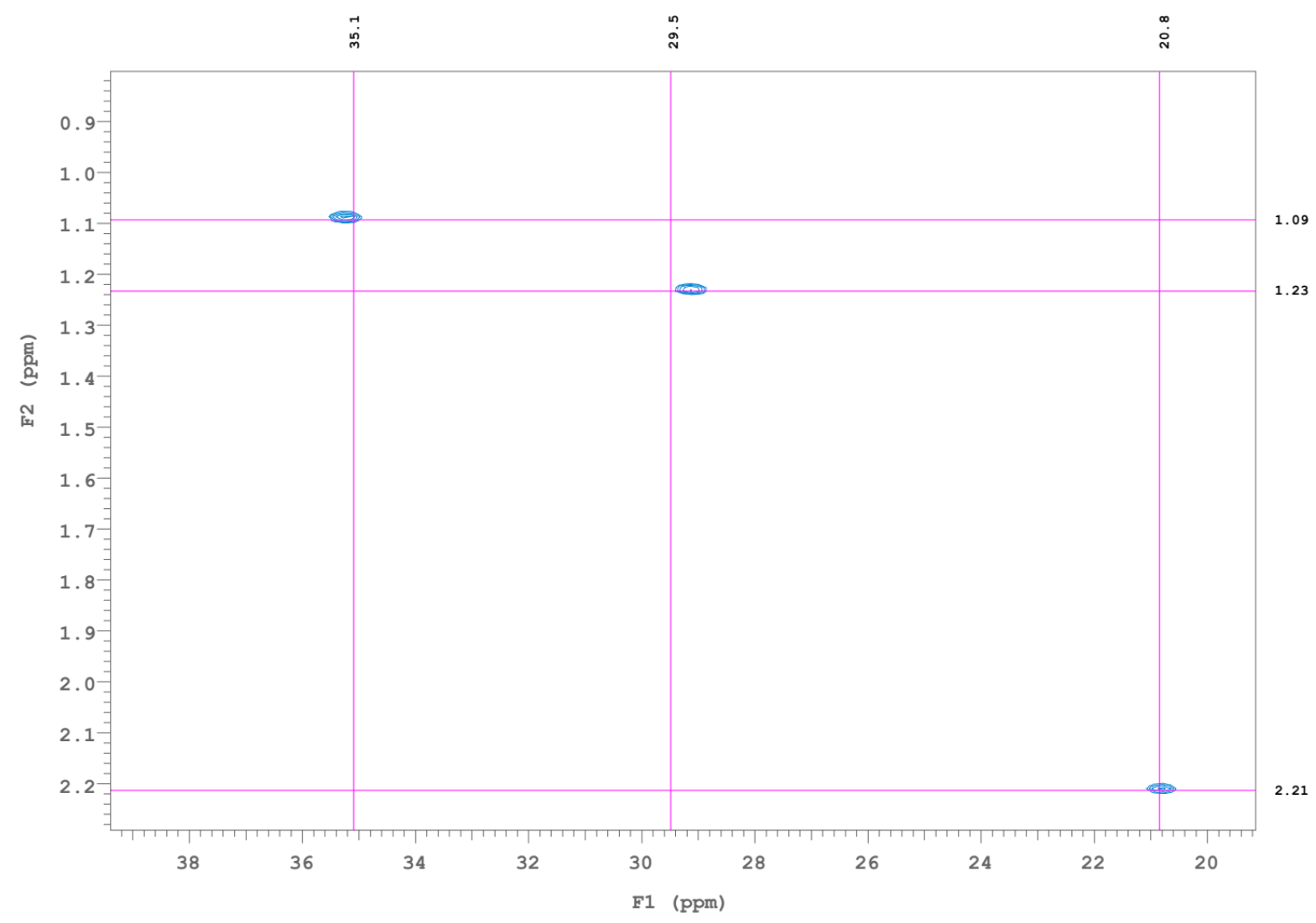

Figure S12. ${ }^{1} \mathrm{H}-{ }^{13} \mathrm{C}$ gHSQC spectrum of $3\left(\mathrm{C}_{6} \mathrm{D}_{6}, 500 \mathrm{MHz}, 25{ }^{\circ} \mathrm{C}\right)$, expanded. 


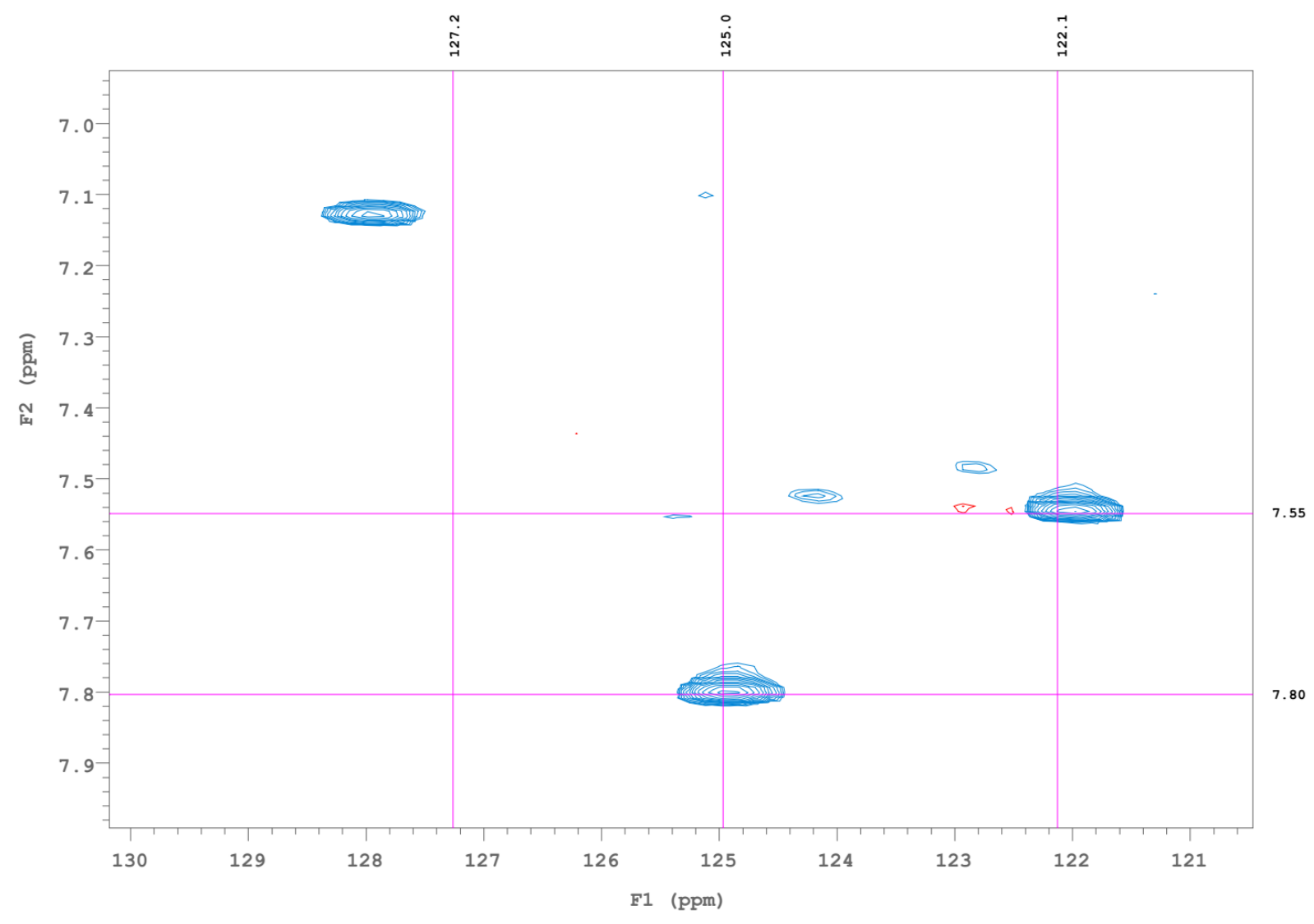

Figure S13. ${ }^{1} \mathrm{H}-{ }^{13} \mathrm{C}$ gHSQC spectrum of $3\left(\mathrm{C}_{6} \mathrm{D}_{6}, 500 \mathrm{MHz}, 25{ }^{\circ} \mathrm{C}\right)$, expanded. 


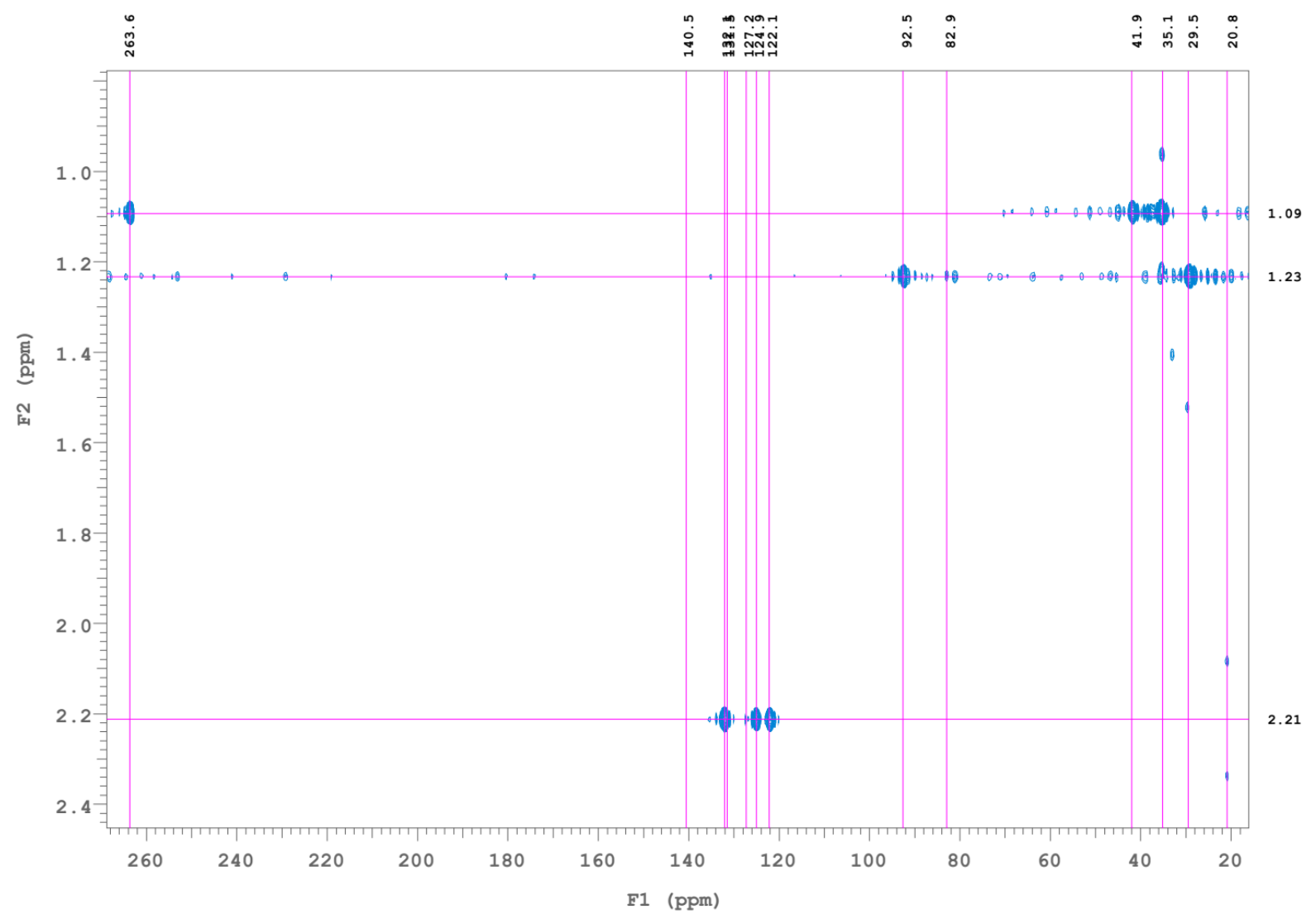

Figure S14. ${ }^{1} \mathrm{H}-{ }^{13} \mathrm{C}$ gHMBC spectrum of $3\left(\mathrm{C}_{6} \mathrm{D}_{6}, 500 \mathrm{MHz}, 25{ }^{\circ} \mathrm{C}\right)$, expanded. 


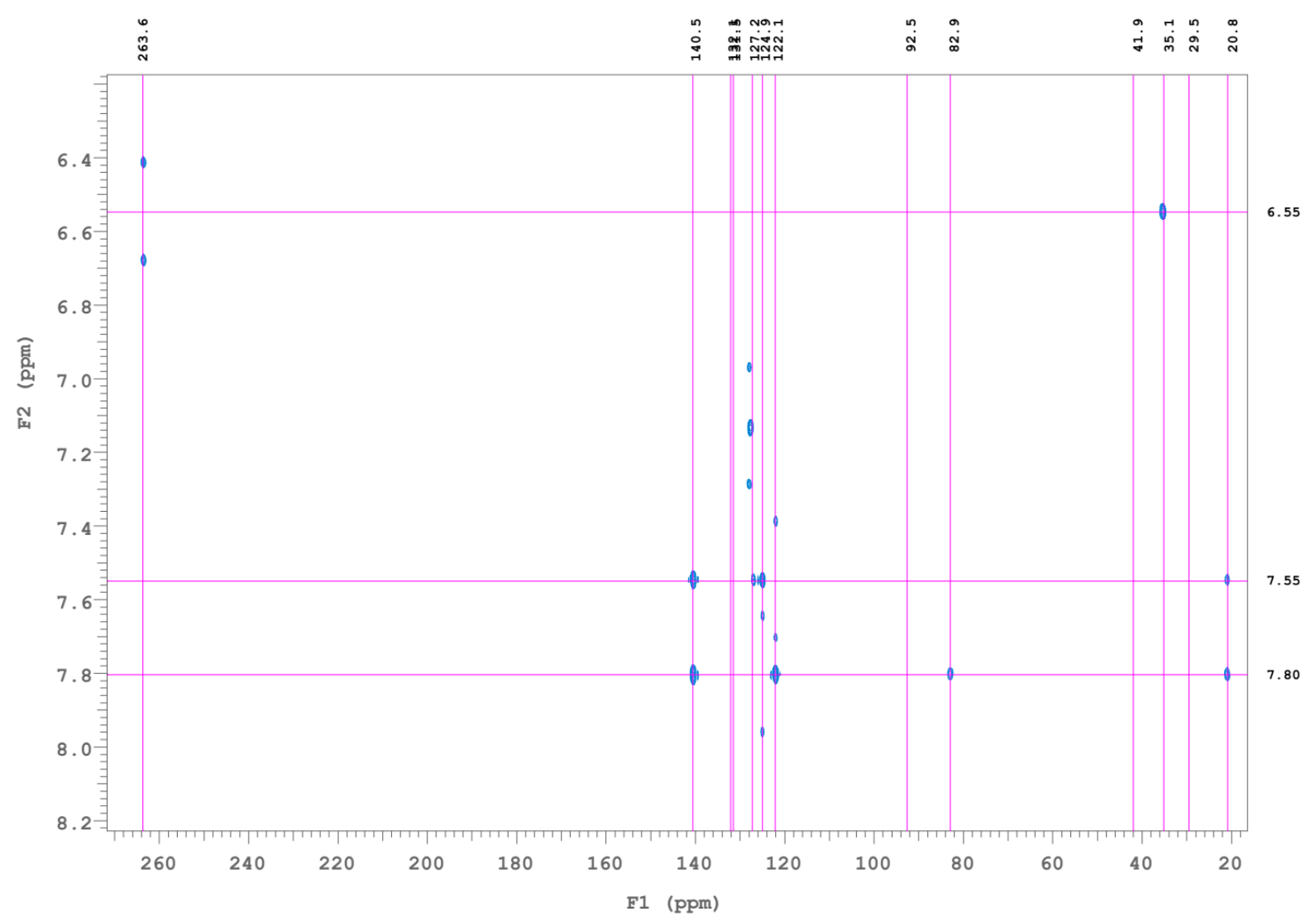

Figure S15. ${ }^{1} \mathrm{H}-{ }^{13} \mathrm{C}$ gHMBC spectrum of $3\left(\mathrm{C}_{6} \mathrm{D}_{6}, 500 \mathrm{MHz}, 25{ }^{\circ} \mathrm{C}\right)$, expanded.

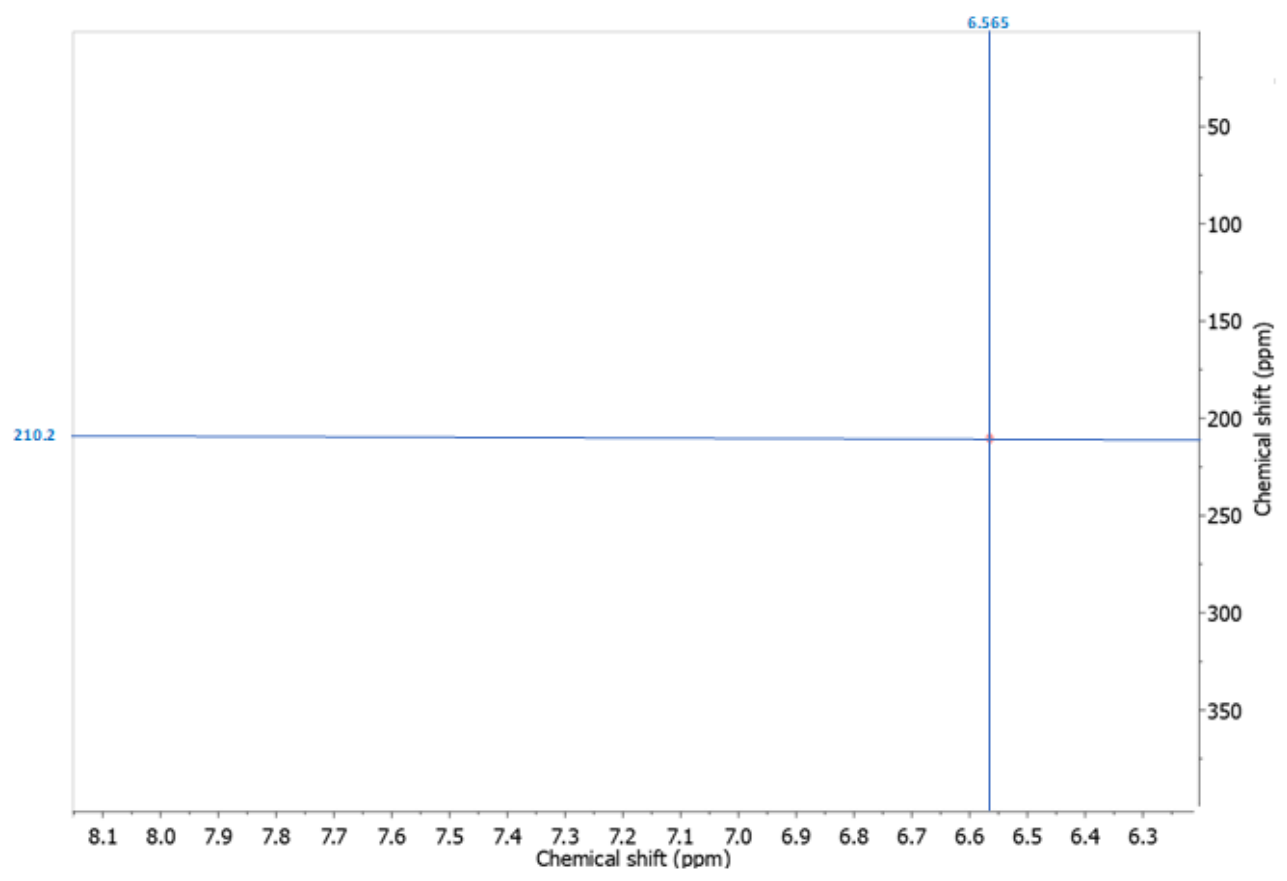

Figure S16. ${ }^{1} \mathrm{H}-{ }^{15} \mathrm{~N}$ gHMBC spectrum of $2\left(\mathrm{C}_{6} \mathrm{D}_{6}, 600 \mathrm{MHz}, 25{ }^{\circ} \mathrm{C}\right)$. 
NMR Characterization of $\left\{\mathrm{CH}_{3} \mathrm{Ph} 3 \mathrm{P}\right\}\left\{[\mathrm{CBZ}-\mathrm{ONO}] \mathrm{W} \equiv \mathrm{C}^{t} \mathrm{Bu}\left(\mathrm{O}^{t} \mathrm{Bu}\right)\right\}$ (4).
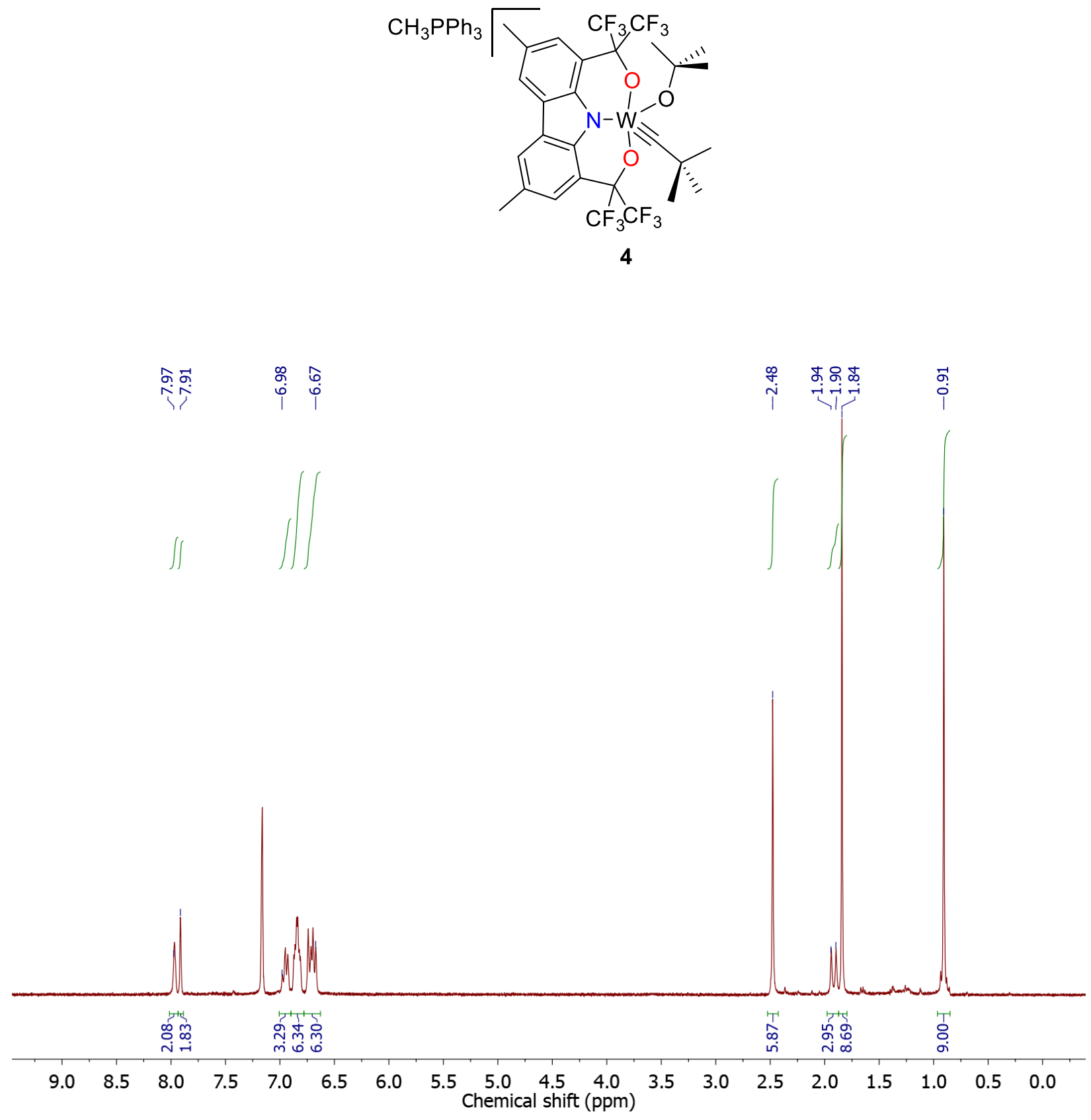

Figure S17. ${ }^{1} \mathrm{H}$ NMR spectrum of $4\left(\mathrm{C}_{6} \mathrm{D}_{6}, 300 \mathrm{MHz}, 25{ }^{\circ} \mathrm{C}\right)$. 


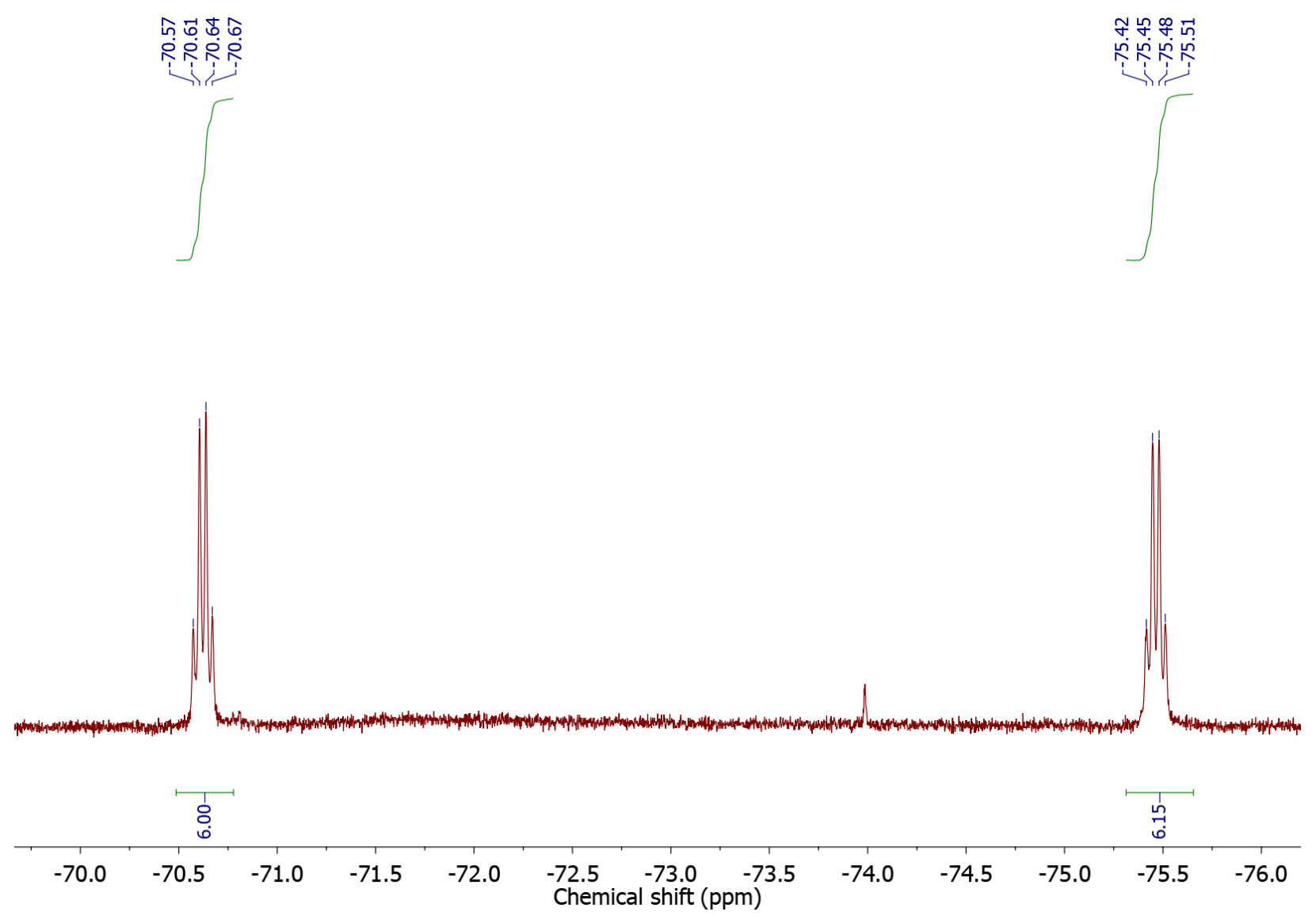

Figure S18. ${ }^{19} \mathrm{~F}$ NMR spectrum of $4\left(\mathrm{C}_{6} \mathrm{D}_{6}, 282 \mathrm{MHz}, 25^{\circ} \mathrm{C}\right)$. 


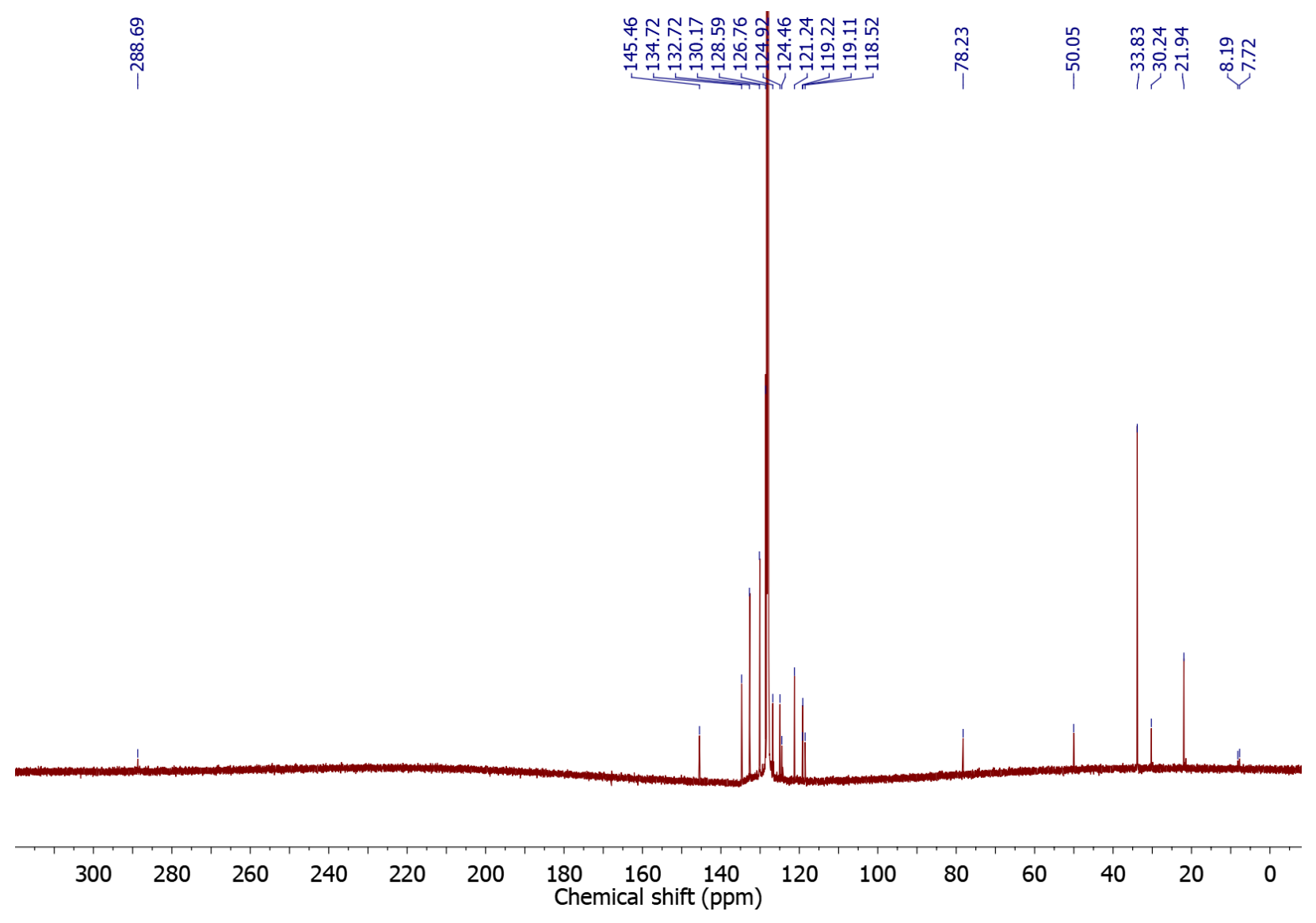

Figure S19. ${ }^{13} \mathrm{C}\left\{{ }^{1} \mathrm{H}\right\}$ NMR spectrum of $4\left(\mathrm{C}_{6} \mathrm{D}_{6}, 125 \mathrm{MHz}, 25{ }^{\circ} \mathrm{C}\right)$. 


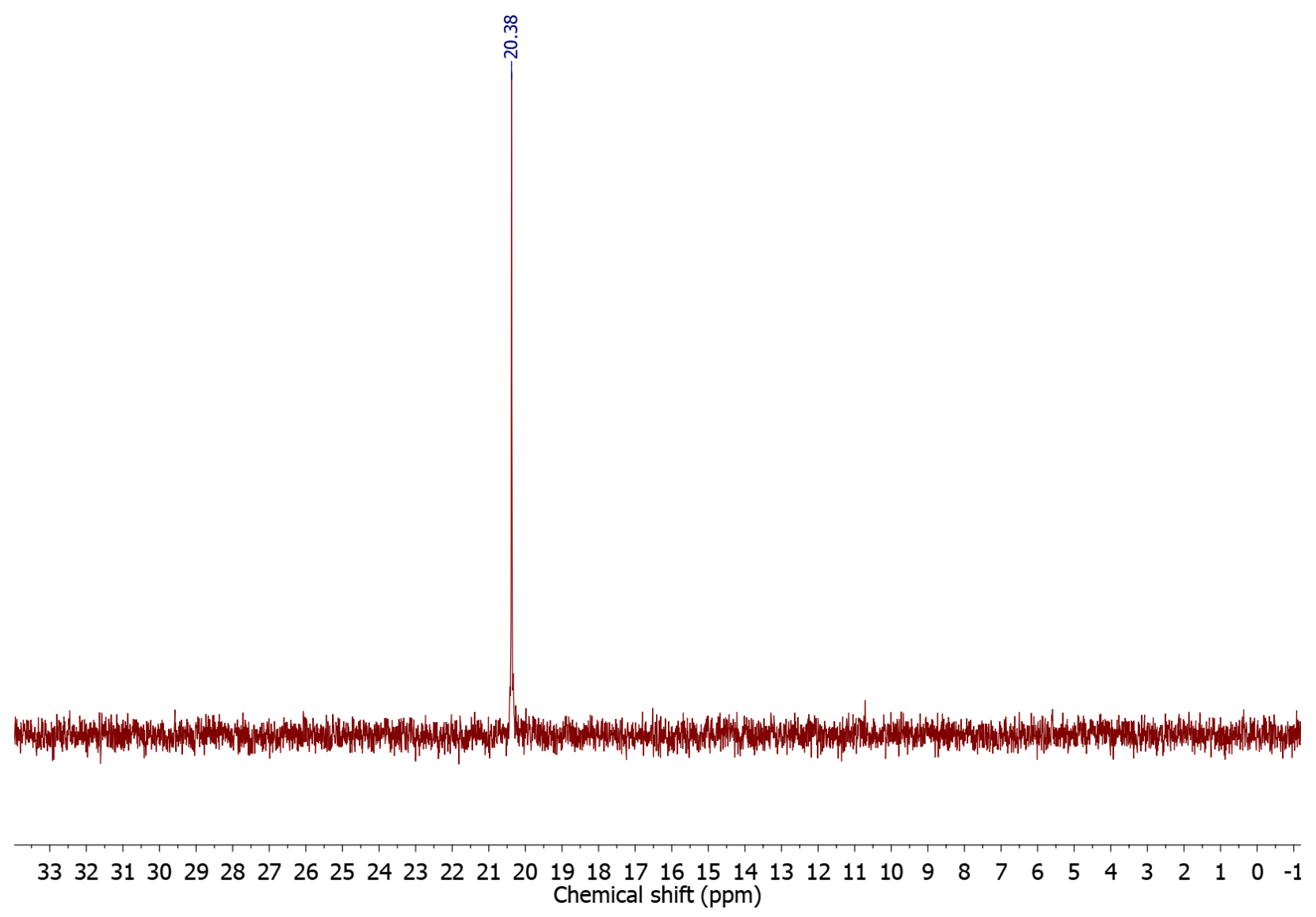

Figure S20. ${ }^{31} \mathrm{P}\left\{{ }^{1} \mathrm{H}\right\}$ NMR spectrum of $4\left(\mathrm{C}_{6} \mathrm{D}_{6}, 121 \mathrm{MHz}, 25{ }^{\circ} \mathrm{C}\right)$. 


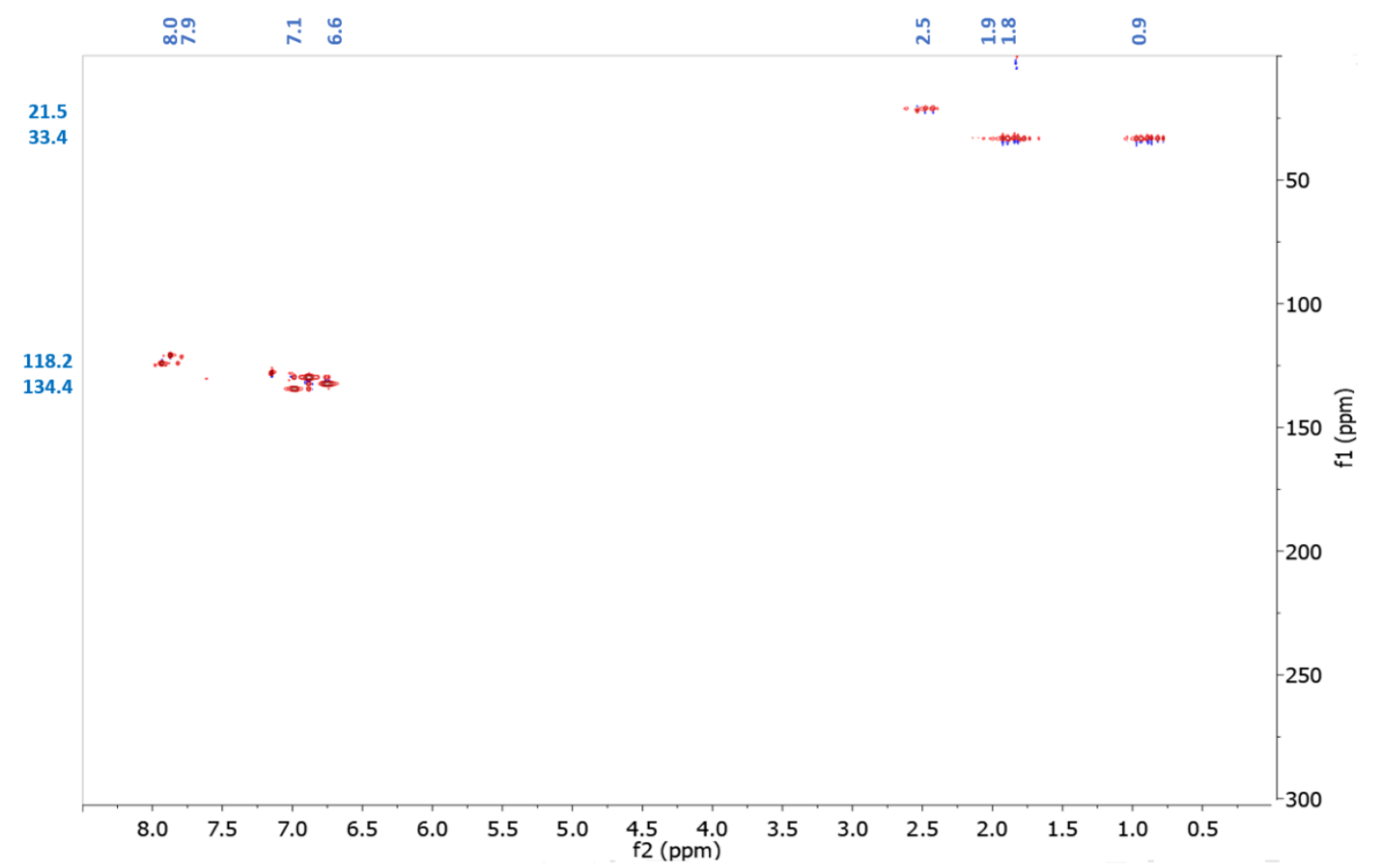

Figure S21. ${ }^{1} \mathrm{H}-{ }^{13} \mathrm{C}$ gHSQC spectrum of $4\left(\mathrm{C}_{6} \mathrm{D}_{6}, 600 \mathrm{MHz}, 25{ }^{\circ} \mathrm{C}\right)$. 


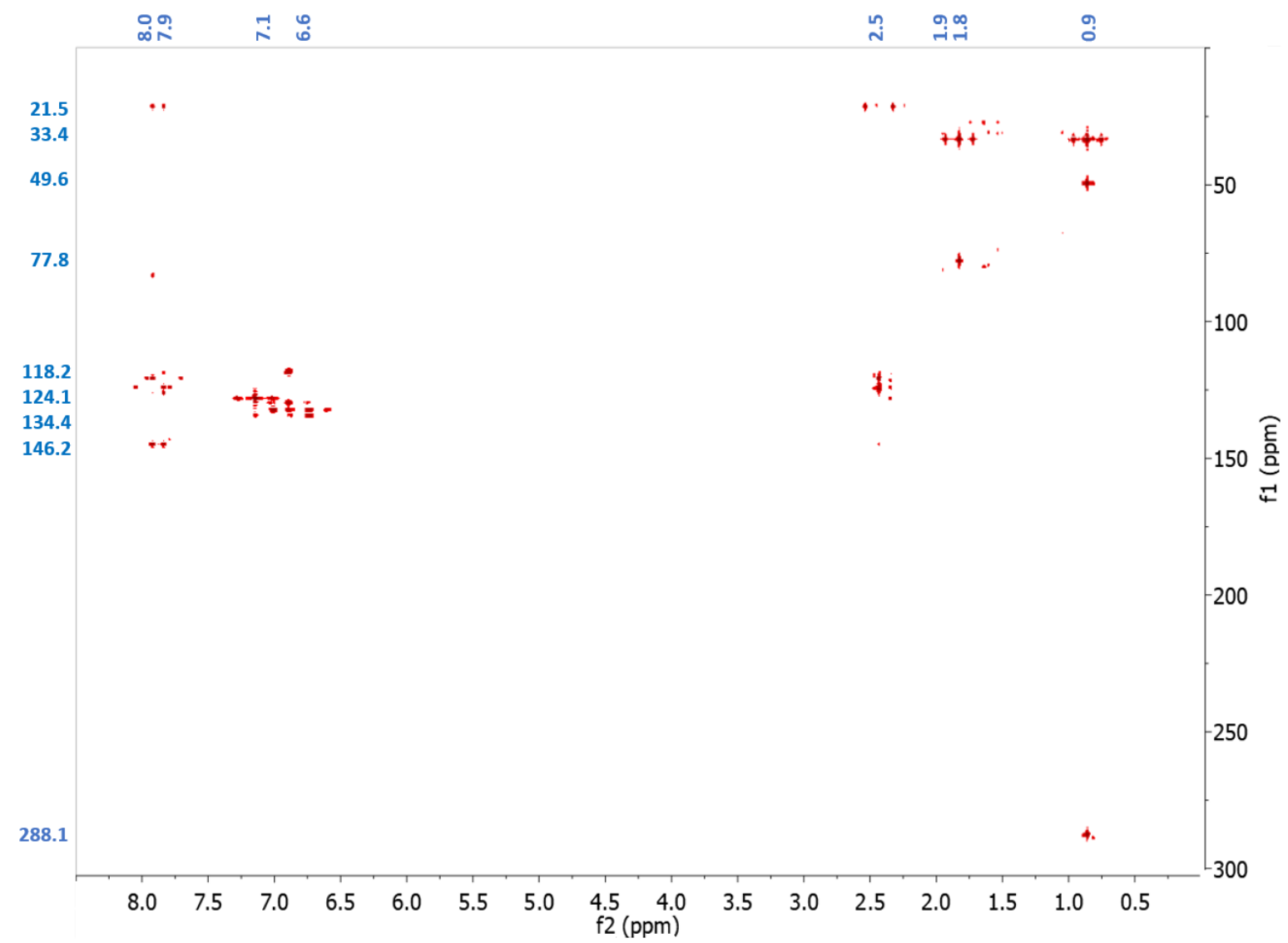

Figure S22. ${ }^{1} \mathrm{H}-{ }^{13} \mathrm{C}$ gHMBC spectrum of $4\left(\mathrm{C}_{6} \mathrm{D}_{6}, 600 \mathrm{MHz}, 25{ }^{\circ} \mathrm{C}\right)$. 
NMR Spectra for the mixture of $[\mathrm{CBZ}-\mathrm{ONO}] \mathrm{W} \equiv \mathrm{O}\left(\mathrm{CH}_{2}{ }^{t} \mathrm{Bu}\right)(6)$ and $[\mathrm{CBZ}$ ONO]W= $=\mathrm{CH}^{t} \mathrm{Bu}\left(\mathrm{O}^{t} \mathrm{Bu}\right)(3)$.

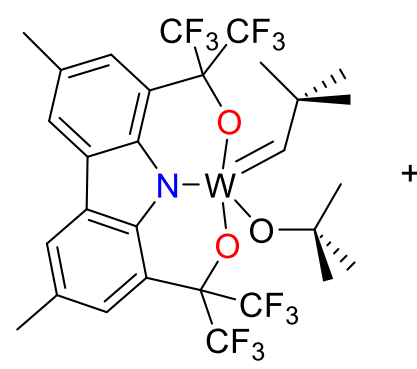

3

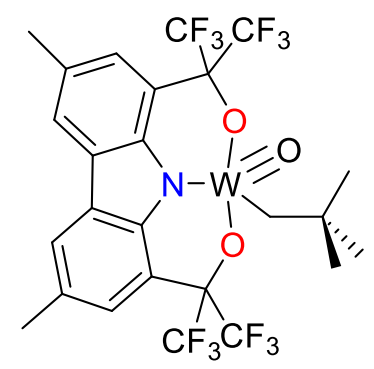

6
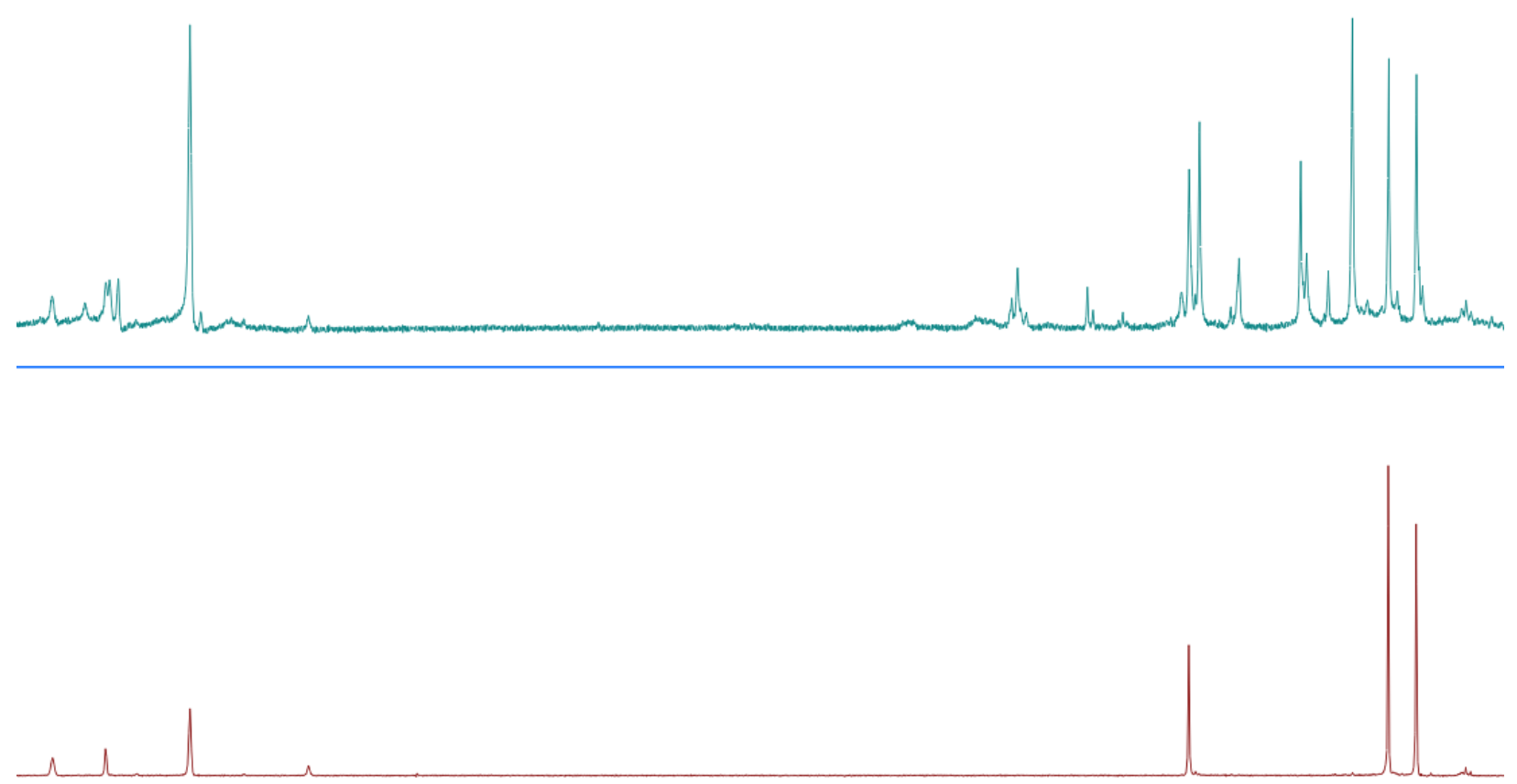

\begin{tabular}{lllllllllllll}
\hline 7.5 & 7.0 & 6.5 & 6.0 & 5.5 & $\begin{array}{c}5.0 \\
\text { Chemical shift (ppm) }\end{array}$ & 4.5 & 4.0 & 3.0 & 2.5 & 2.0 & 1.5 & 1.0
\end{tabular}

Figure S23. ${ }^{1} \mathrm{H}$ NMR stack plot of $\mathbf{3}$ and the mixture of $\mathbf{6}+\mathbf{3}\left(\mathrm{C}_{6} \mathrm{D}_{6}, 300 \mathrm{MHz}, 25^{\circ} \mathrm{C}\right.$, Green: mixture of $\mathbf{6}+\mathbf{3}$, Red: $\mathbf{3}$ ). 


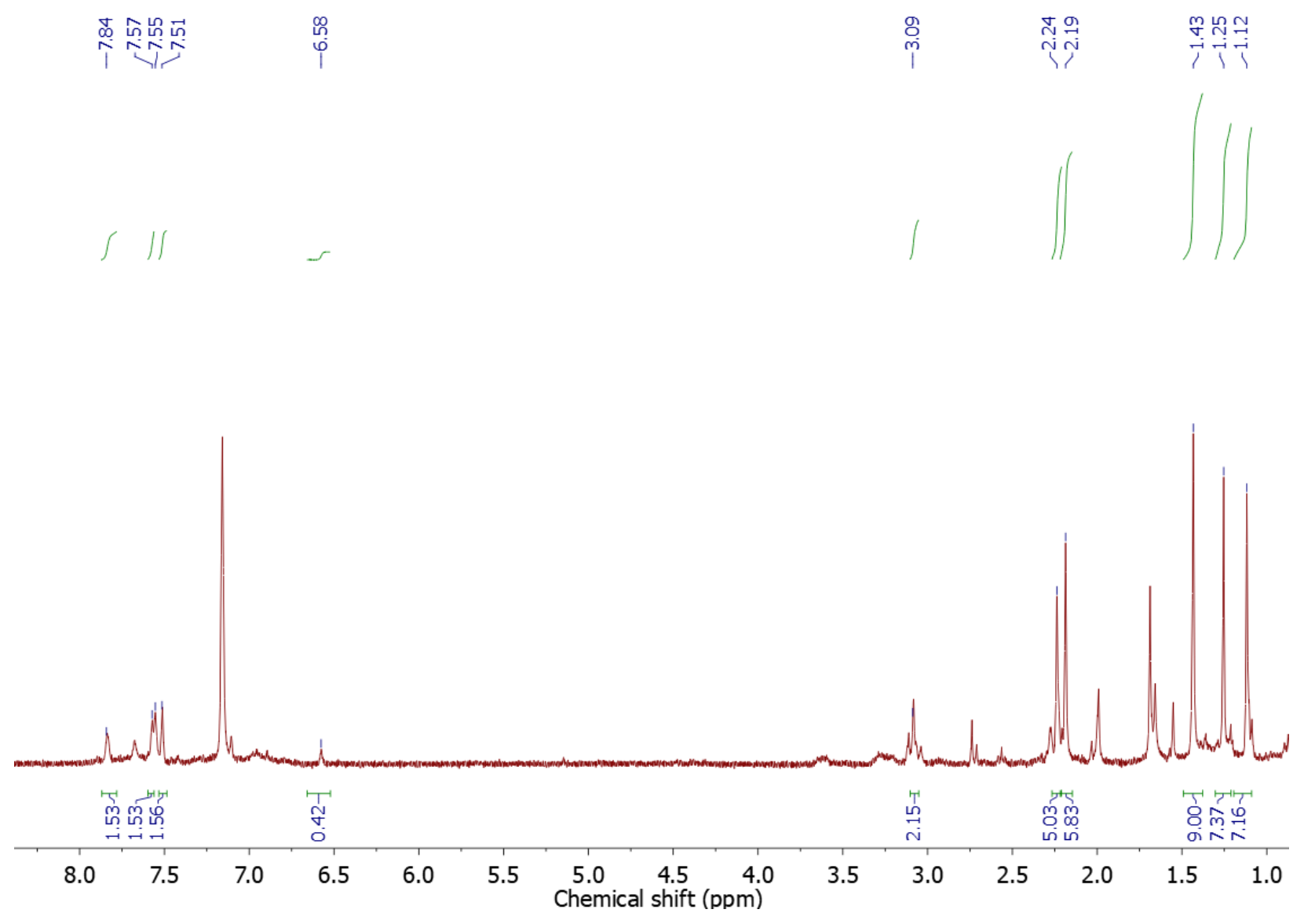

Figure S24. ${ }^{1} \mathrm{H}$ NMR spectrum of 6 in a mixture with $3\left(\mathrm{C}_{6} \mathrm{D}_{6}, 300 \mathrm{MHz}, 25^{\circ} \mathrm{C}\right)$. 

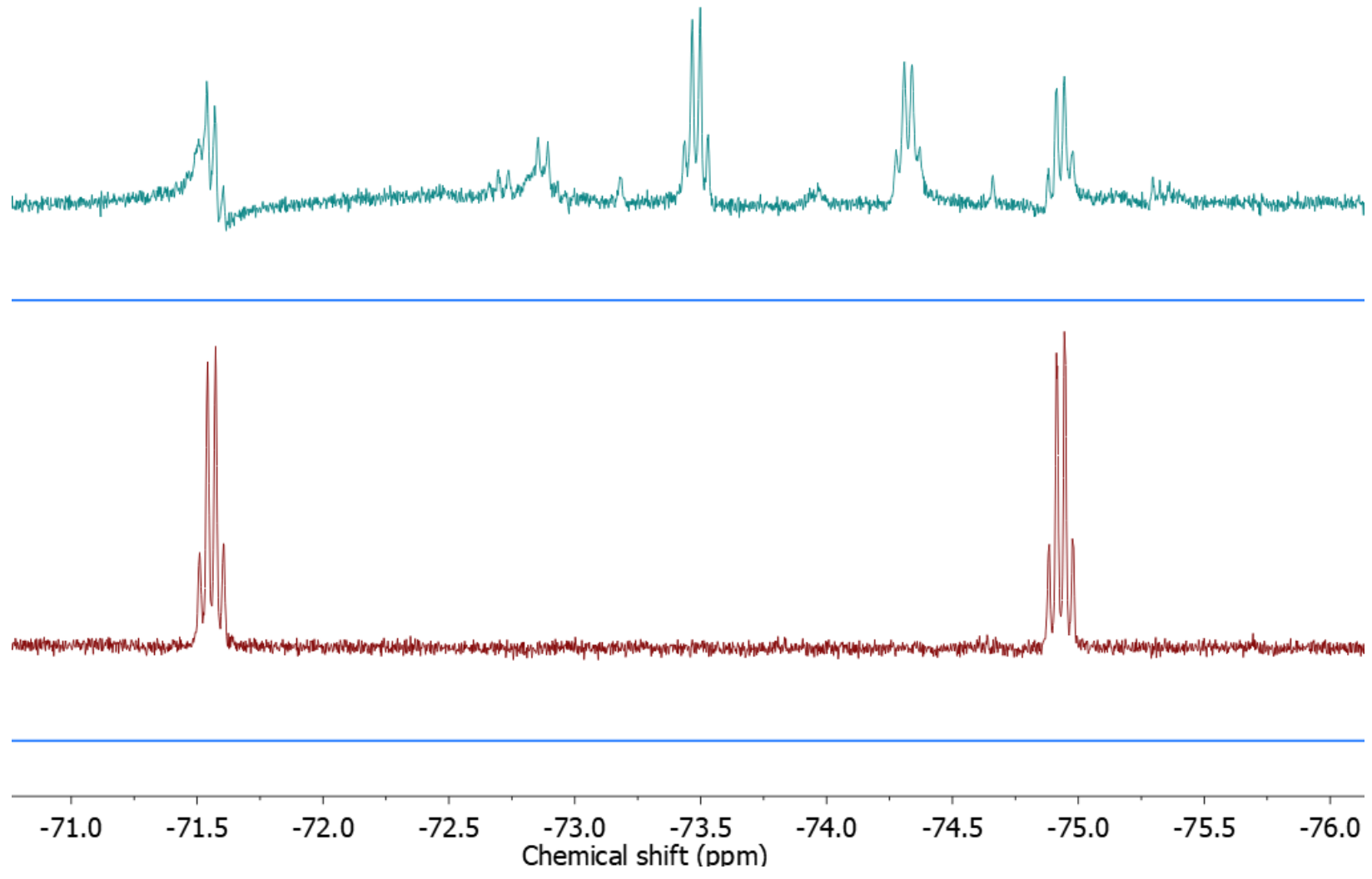

Figure S25. ${ }^{19} \mathrm{~F}$ NMR stack plot of $\mathbf{3}$ and the mixture of $\mathbf{6}+\mathbf{3}\left(\mathrm{C}_{6} \mathrm{D}_{6}, 282 \mathrm{MHz}, 25{ }^{\circ} \mathrm{C}\right.$, Green: mixture of $\mathbf{6}+\mathbf{3}$, Red: $\mathbf{3}$ ). 


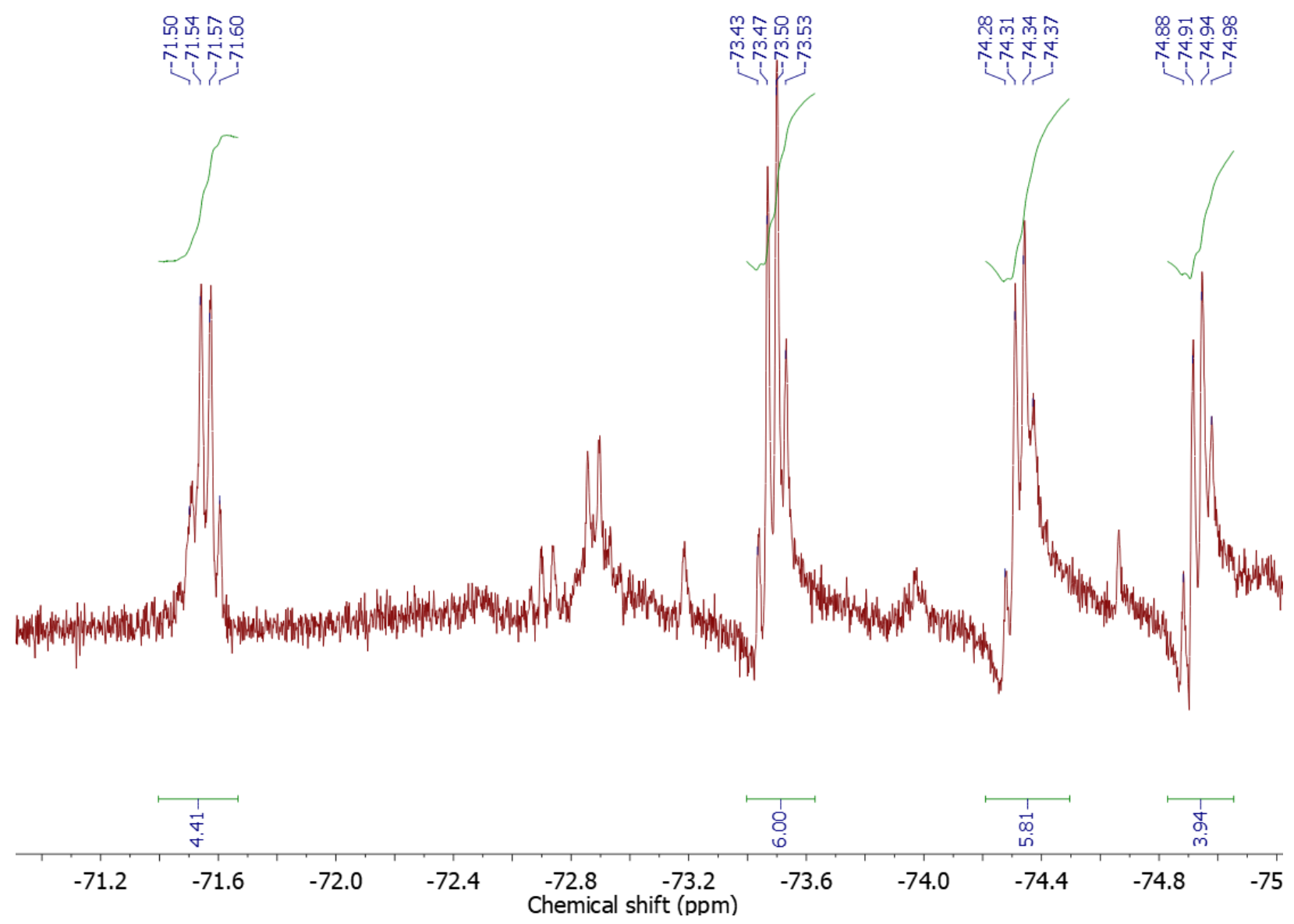

Figure S26. ${ }^{19} \mathrm{~F}$ NMR spectrum of 6 in a mixture with $3\left(\mathrm{C}_{6} \mathrm{D}_{6}, 282 \mathrm{MHz}, 25^{\circ} \mathrm{C}\right)$. 
NMR spectra for the in situ generation of $[\mathrm{CBZ}-\mathrm{ONO}] \mathrm{W} \equiv \mathrm{O}\left(\mathrm{CH}_{2}{ }^{t} \mathrm{Bu}\right)(6)$ and isobutylene expulsion.
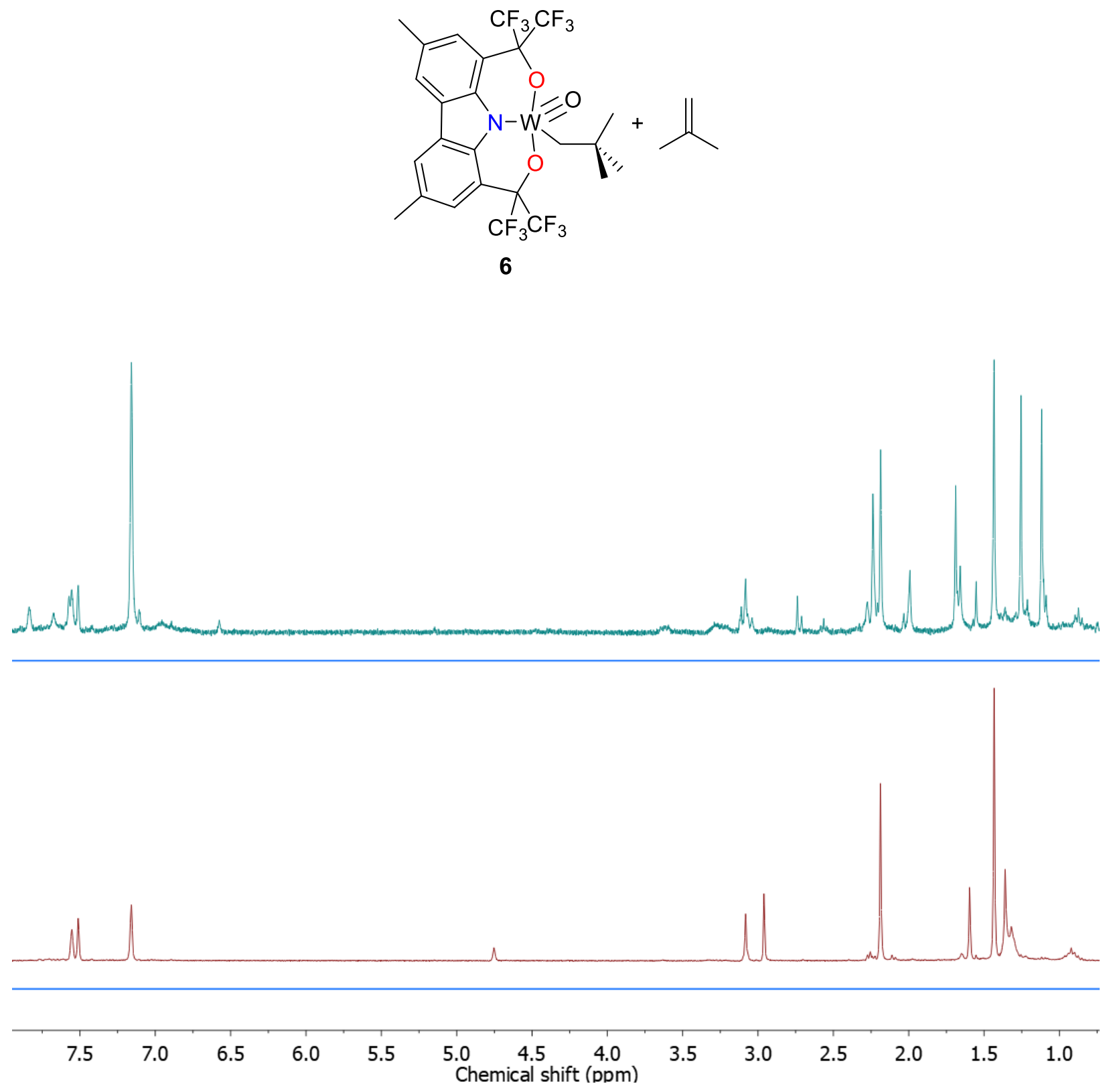

Figure S27. ${ }^{1} \mathrm{H}$ NMR stack plot for in situ generation of $\mathbf{6}$ and the mixture of $\mathbf{6}+\mathbf{3}\left(\mathrm{C}_{6} \mathrm{D}_{6}, 300\right.$ $\mathrm{MHz}, 25^{\circ} \mathrm{C}$, Green: mixture of $\mathbf{6}+\mathbf{3}$, Red: in situ generation of $\mathbf{6}$ ). 


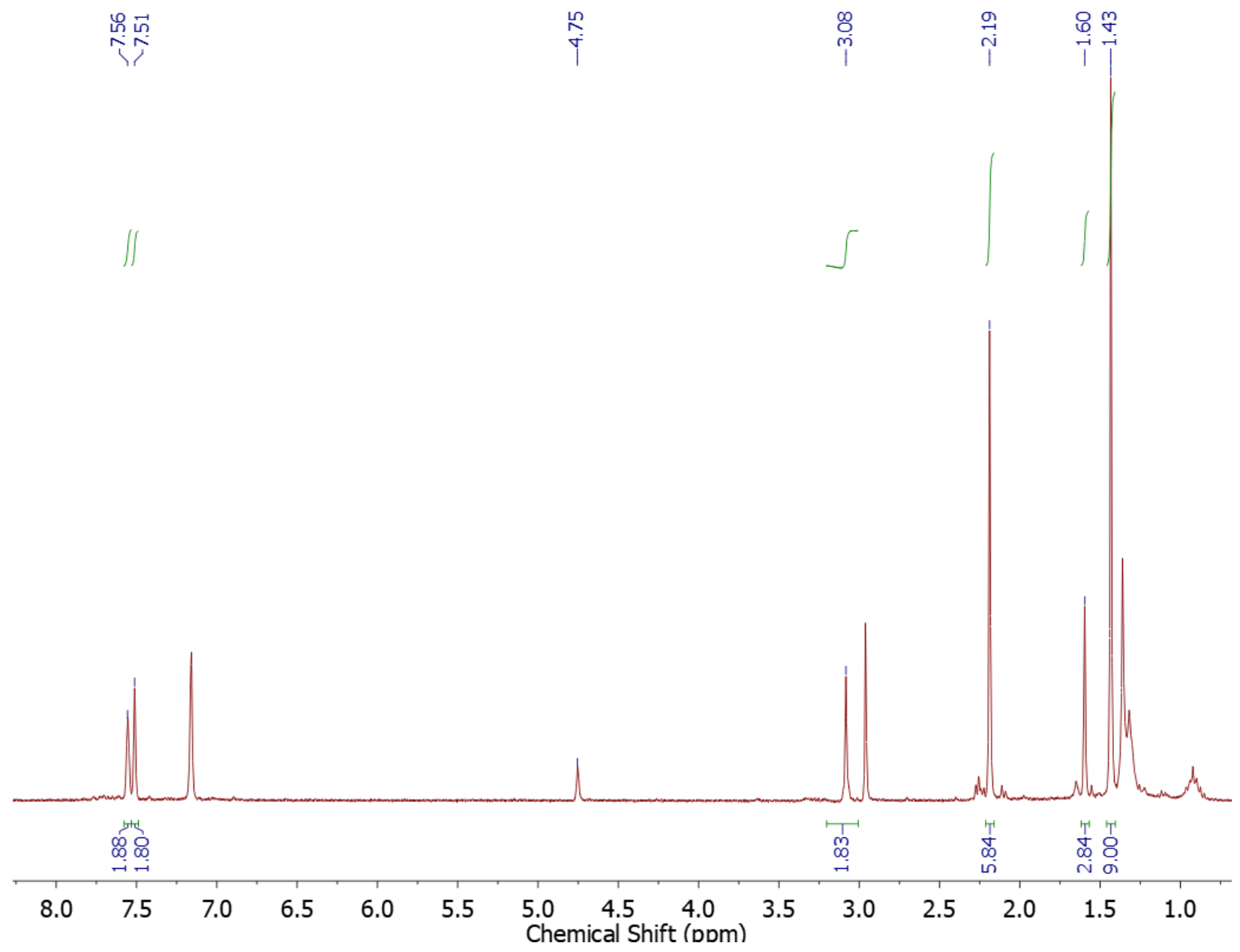

Figure S28. ${ }^{1} \mathrm{H}$ NMR spectrum for in situ generation 6 with isobutylene expulsion $\left(\mathrm{C}_{6} \mathrm{D}_{6}, 300\right.$ $\left.\mathrm{MHz}, 25^{\circ} \mathrm{C}\right)$. 

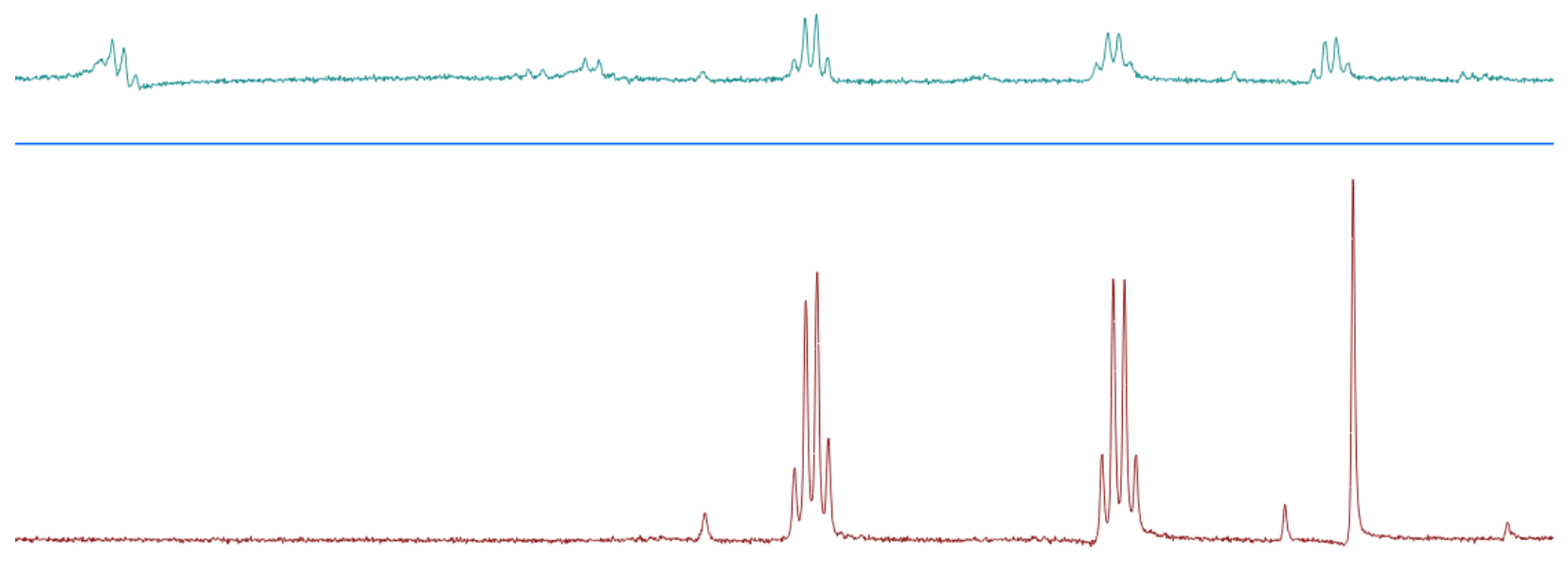

$-71.4-71.6-71.8-72.0-72.2-72.4-72.6-72.8-73.0-73.2-73.4-73.6-73.8-74.0-74.2-74.4-74.6-74.8-75.0-75.2-75.4$ Chemical shift (ppm)

Figure S29. ${ }^{19} \mathrm{~F}$ NMR stack plot for in situ generation of $\mathbf{6}$ and the mixture of $\mathbf{6}+\mathbf{3}\left(\mathrm{C}_{6} \mathrm{D}_{6}, 282\right.$ $\mathrm{MHz}, 25^{\circ} \mathrm{C}$, Green: mixture of $\mathbf{6}+\mathbf{3}$, Red: in situ generation of $\mathbf{6}$ with signal from MeOTf). 


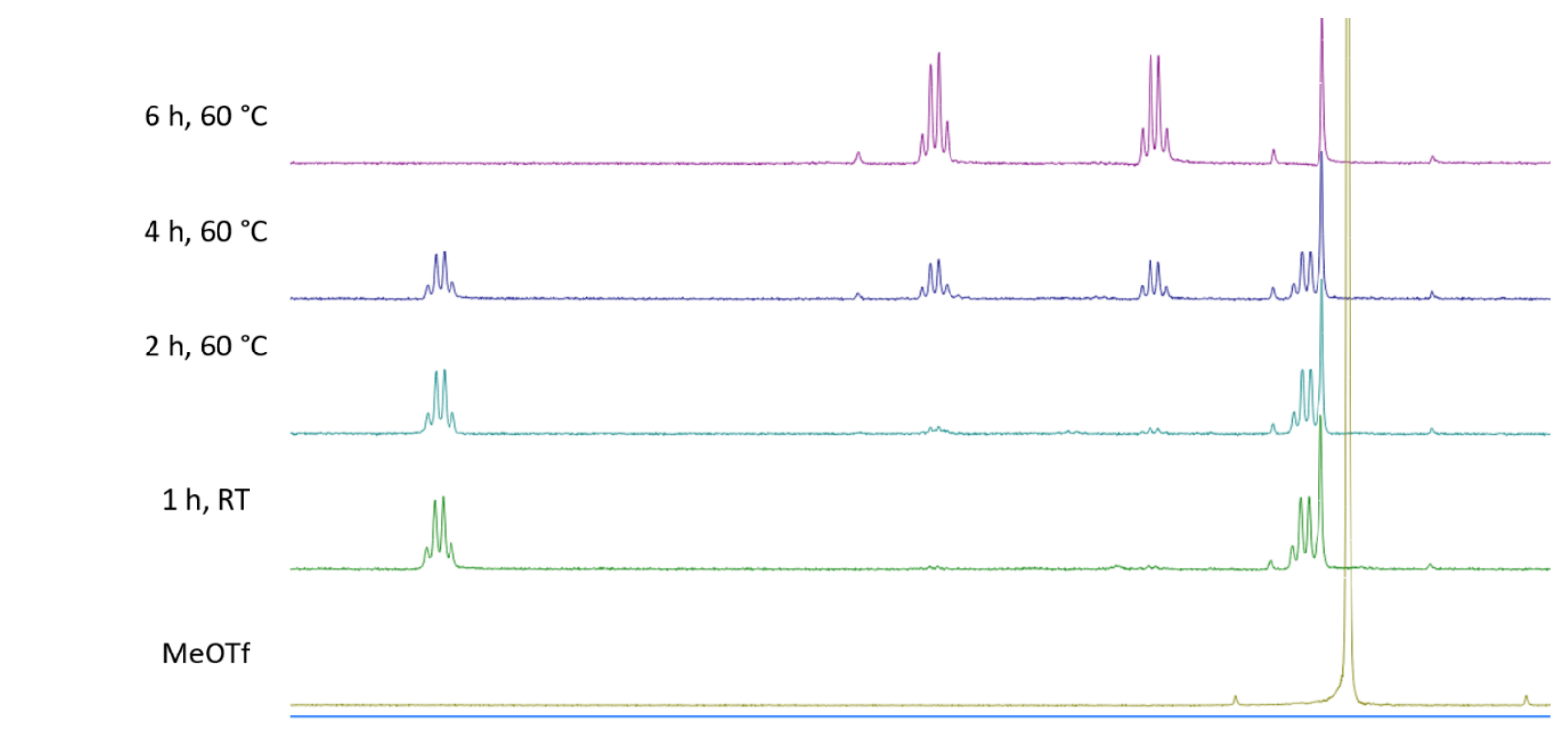

$[\mathrm{CBZ}-\mathrm{ONO}] \mathrm{W}=\mathrm{CH}^{\mathrm{t} B u}\left(\mathrm{O}^{\mathrm{t}} \mathrm{Bu}\right)(\mathbf{3})$

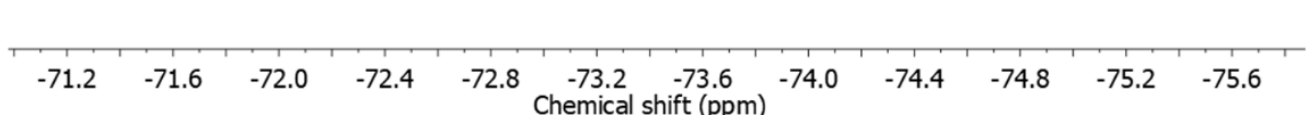

Figure S30. ${ }^{19} \mathrm{~F}$ NMR spectra series of the NMR tube reaction between [CBZ$\mathrm{ONO}] \mathrm{W}=\mathrm{CH}^{t} \mathrm{Bu}\left(\mathrm{O}^{t} \mathrm{Bu}\right)(3)$ and $\mathrm{MeOTf}\left(\mathrm{C}_{6} \mathrm{D}_{6}, 282 \mathrm{MHz}, 25^{\circ} \mathrm{C}\right.$, in situ generation of [CBZ$\mathrm{ONO}] \mathrm{W} \equiv \mathrm{O}\left(\mathrm{CH}_{2}{ }^{t} \mathrm{Bu}\right)(\mathbf{6})$ is evident from the blue spectrum taken after $4 \mathrm{~h}$ of heating the NMR tube at $\left.60{ }^{\circ} \mathrm{C}\right)$. 


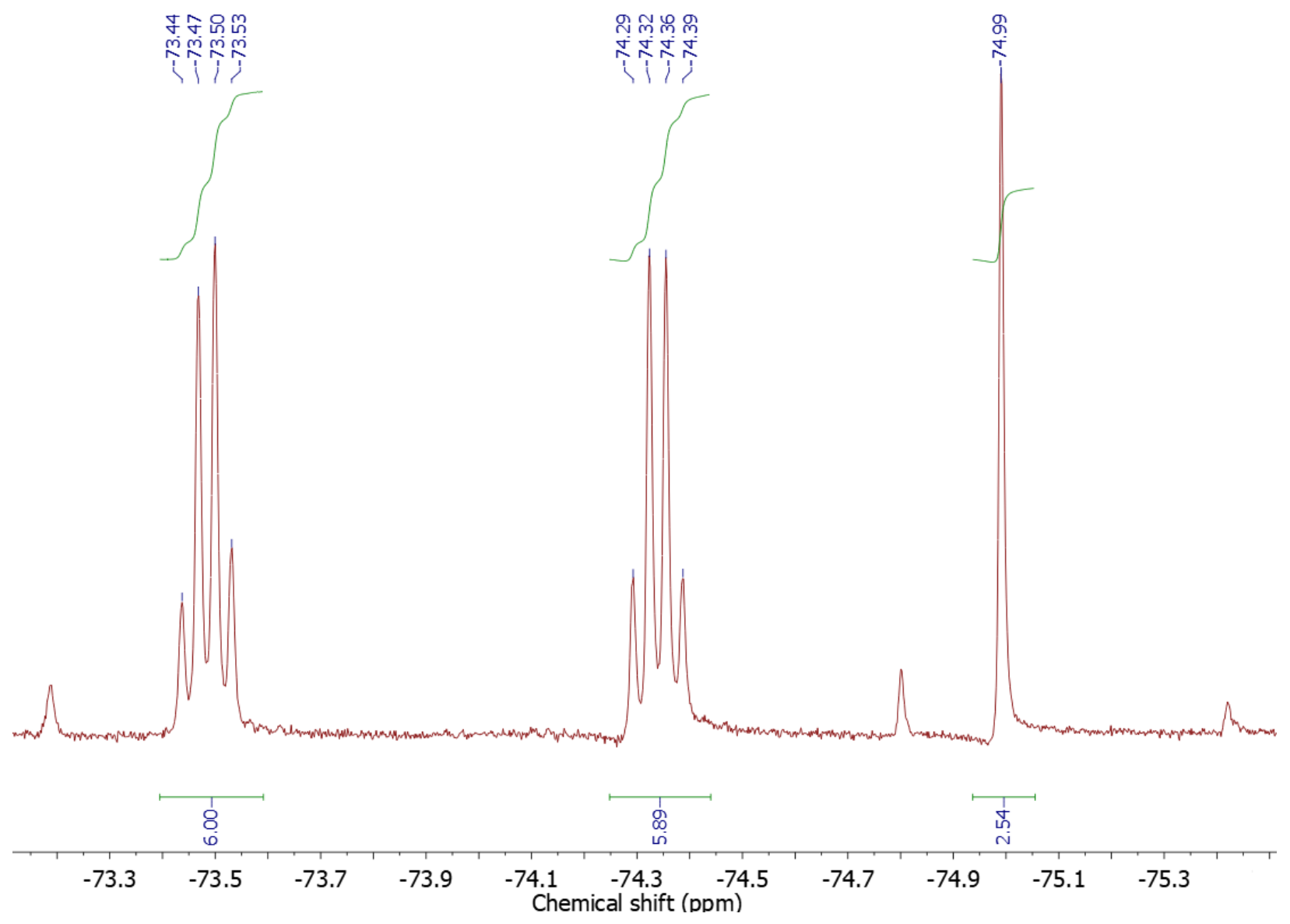

Figure S31. ${ }^{19} \mathrm{~F}$ NMR spectrum for in situ generation 6 with signal from MeOTf $\left(\mathrm{C}_{6} \mathrm{D}_{6}, 282\right.$ $\left.\mathrm{MHz}, 25^{\circ} \mathrm{C}\right)$. 
$\underline{\text { UV-Vis Study }}$

UV-Vis Characterization of $\left\{\mathrm{CH}_{3} \mathrm{Ph}_{3} \mathrm{P}\right\}\left\{[\mathrm{CBZ}-\mathrm{ONO}] \mathrm{W} \equiv \mathrm{C}^{t} \mathrm{Bu}\left(\mathrm{O}^{t} \mathrm{Bu}\right)\right\}$ (4).

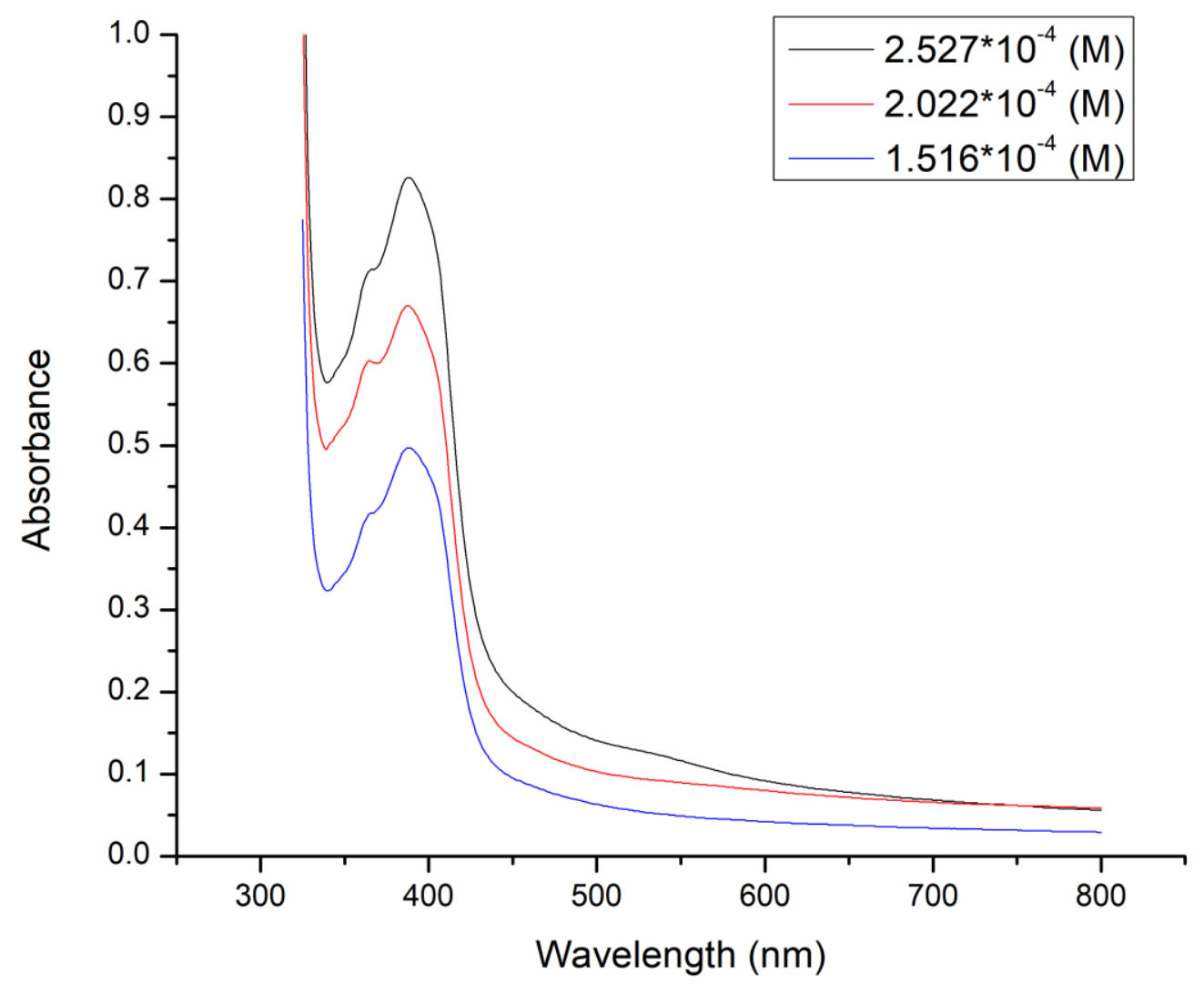

Figure S32. UV-Vis spectrum of 4 for 3 different concentrations $\left(\mathrm{C}_{6} \mathrm{H}_{6}, 25^{\circ} \mathrm{C}\right), \lambda_{\max }$ at 389.4 $\mathrm{nm}$. 


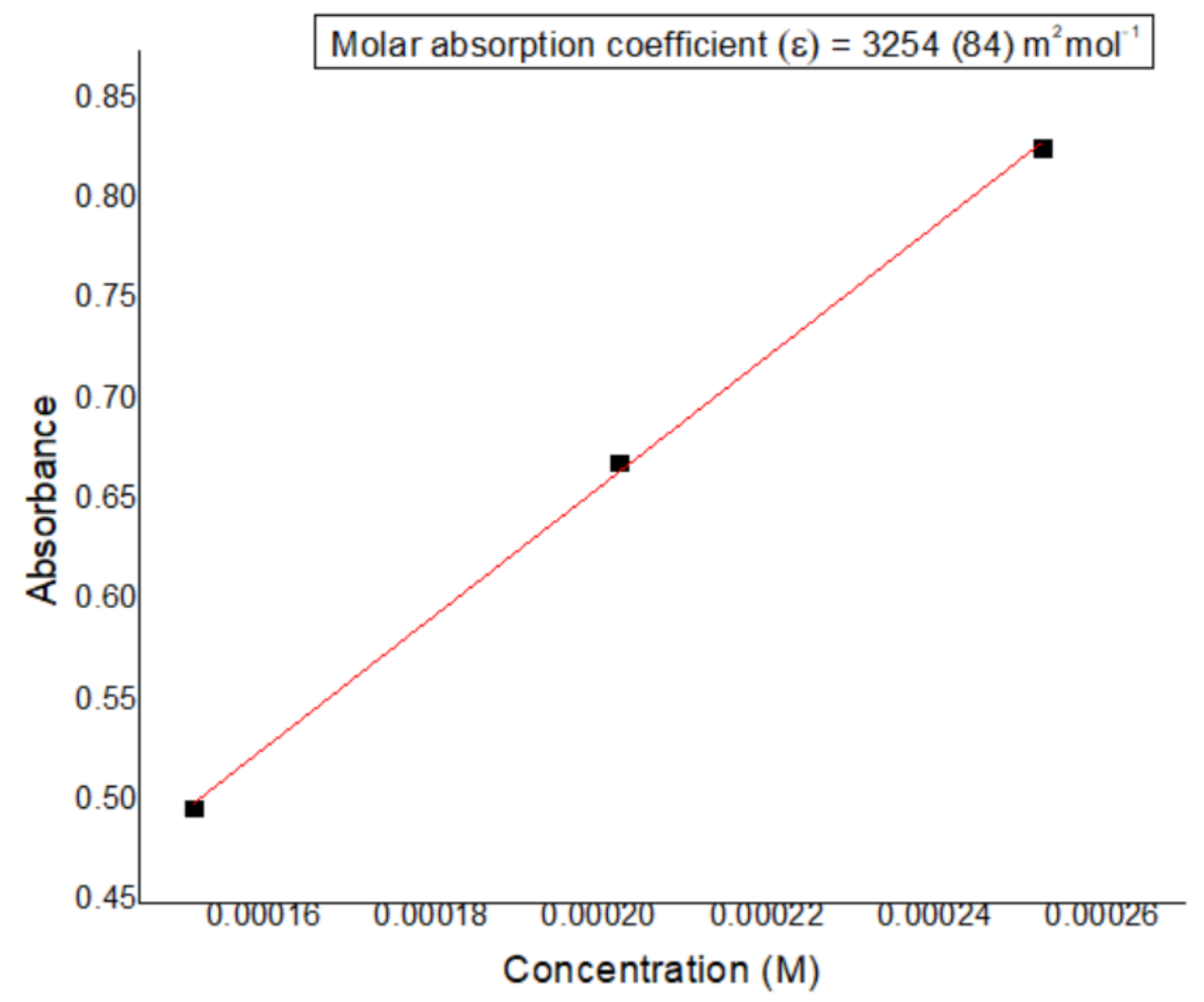

Figure S33. Molar absorption coefficient of $4\left(\mathrm{C}_{6} \mathrm{H}_{6}, 25^{\circ} \mathrm{C}\right)$. 
NMR Characterization of $\left\{\mathrm{CH}_{3} \mathrm{Ph}_{3} \mathrm{P}\right\}\left\{\left[\mathrm{CF}_{3}-\mathrm{ONO}\right] \mathrm{W} \equiv \mathrm{C}^{t} \mathrm{Bu}\left(\mathrm{O}^{t} \mathrm{Bu}\right)\right\}(7){ }^{5}$
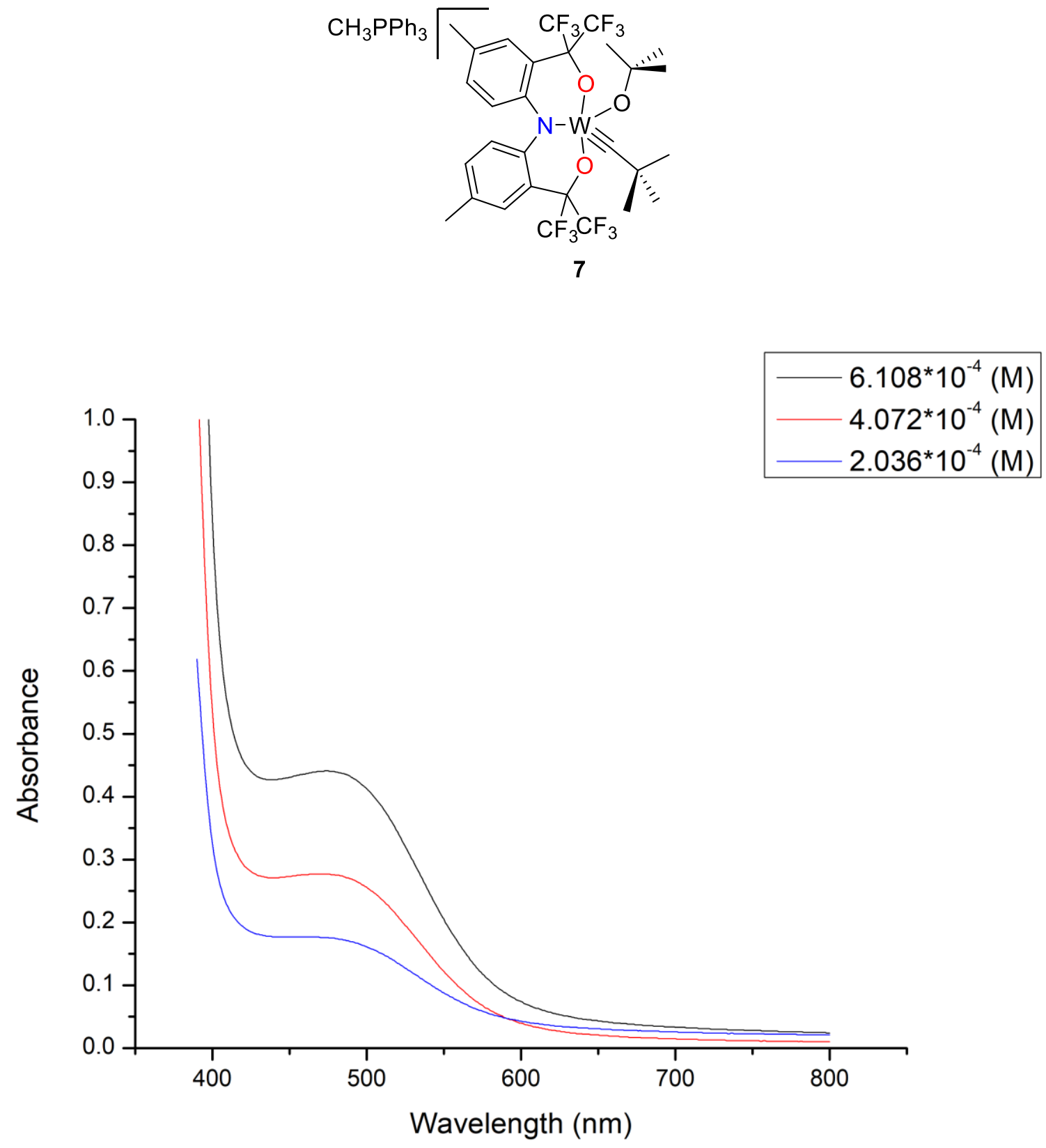

Figure S34. UV-Vis spectrum of 7 for 3 different concentrations $\left(\mathrm{C}_{6} \mathrm{H}_{6}, 25^{\circ} \mathrm{C}\right), \lambda_{\max }$ at 478.3 nm. 


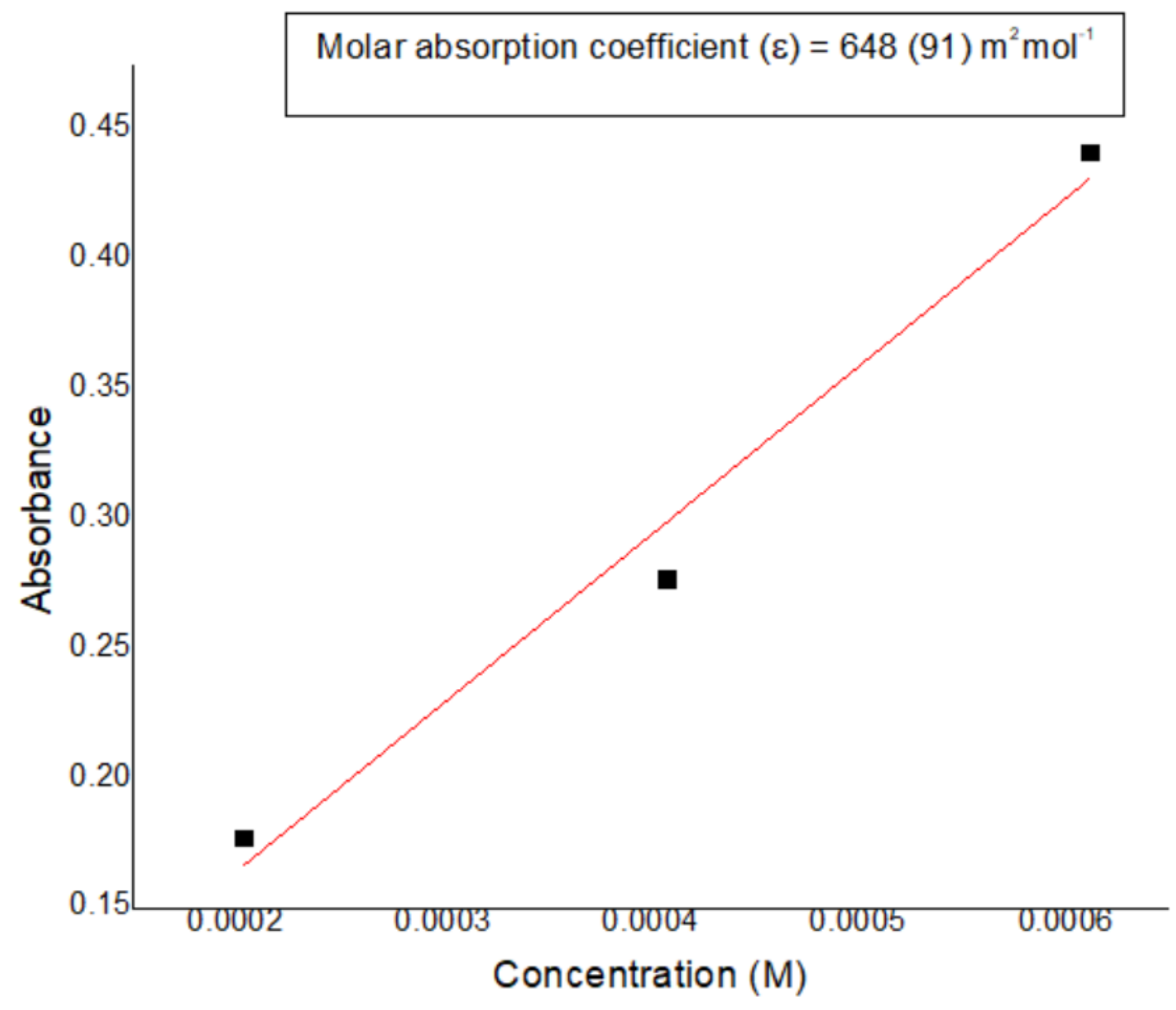

Figure S35. Molar absorption coefficient of $7\left(\mathrm{C}_{6} \mathrm{H}_{6}, 25^{\circ} \mathrm{C}\right)$. 


\section{$\underline{\text { X-Ray Crystallography of } 3}$}

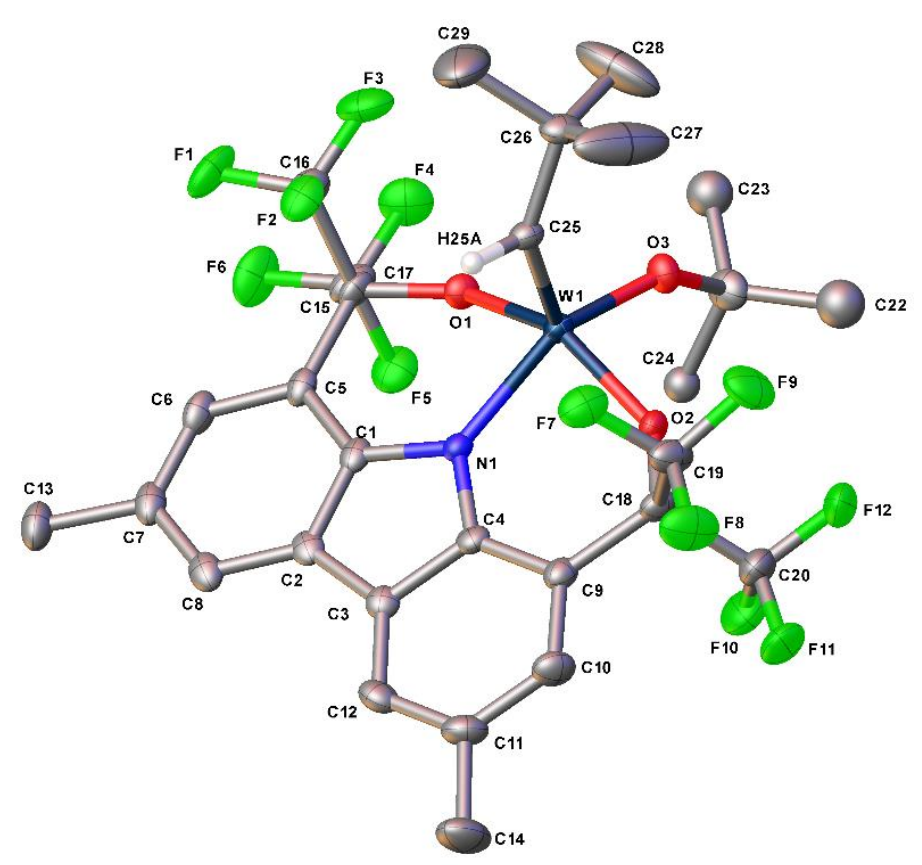

Figure S36. Molecular structure of $\mathbf{3}$.

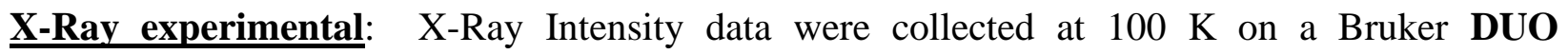
diffractometer using MoK $\alpha$ radiation $(\lambda=0.71073 \AA)$ and an APEXII CCD area detector.

Raw data frames were read by program $\mathrm{SAINT}^{7}$ and integrated using 3D profiling algorithms. The resulting data were reduced to produce hkl reflections and their intensities and estimated standard deviations. The data were corrected for Lorentz and polarization effects and numerical absorption corrections were applied based on indexed and measured faces.

The structure was solved and refined in SHELXTL2014, ${ }^{8,9}$ using full-matrix least-squares refinement. The non- $\mathrm{H}$ atoms were refined with anisotropic thermal parameters and all of the $\mathrm{H}$ atoms were calculated in idealized positions and refined riding on their parent atoms. The asymmetric unit consists of two molecules that are chemically equivalent but crystallographically independent. The first molecule has the methyl groups on $\mathrm{C} 21$ disordered and refined in two parts. 
The second molecule has significantly disordered regions. All Fluorine atoms of $\mathrm{CF}_{3}$ are disordered and in each case were resolved into two parts refined dependently. The last disorders are in the O-t-butyl and C-t-butyl switching places and causing all methyl groups to be disordered. In the final cycle of refinement, 14,194 reflections (of which 11,063 are observed with I > $2 \sigma(\mathrm{I})$ ) were used to refine 808 parameters and the resulting $\mathrm{R}_{1}, \mathrm{wR}_{2}$ and $\mathrm{S}$ (goodness of fit) were $3.95 \%$, $9.58 \%$ and 1.050 , respectively. The refinement was carried out by minimizing the $\mathrm{wR}_{2}$ function using $\mathrm{F}^{2}$ rather than $\mathrm{F}$ values. $\mathrm{R}_{1}$ is calculated to provide a reference to the conventional $\mathrm{R}$ value but its function is not minimized.

The structure is re-refined with anisotropic displacement parameters for all atoms, and all site occupation factors of disordered parts are dependently refined. Additionally, the displacement parameters of similar atoms were re/con-strained to maintain similar anisotropic displacement parameters.

SHELXTL2014 (2014). Bruker-AXS, Madison, Wisconsin, USA.

Table S1. Crystal data and structure refinement for ush2.

Identification code

Empirical formula

Formula weight

Temperature

Wavelength

Crystal system

Space group

Unit cell dimensions

Volume

Z

Density (calculated)

Absorption coefficient ush2

C29 H29 F12 N O3 W

851.38

100(2) K

$0.71073 \AA$

Monoclinic

$\mathrm{P} 21 / \mathrm{n}$

$\mathrm{a}=22.5913(8) \AA \quad \alpha=90^{\circ}$.

$\mathrm{b}=11.3476(4) \AA \quad \beta=95.9400(10)^{\circ}$.

$\mathrm{c}=24.2179(8) \AA \quad \gamma=90^{\circ}$.

6175.1(4) $\AA^{3}$

8

$1.832 \mathrm{Mg} / \mathrm{m}^{3}$

$3.846 \mathrm{~mm}^{-1}$ 
$\mathrm{F}(000)$

Crystal size

Theta range for data collection

Index ranges

Reflections collected

Independent reflections

Completeness to theta $=25.000^{\circ}$

Absorption correction

Max. and min. transmission

Refinement method

Data / restraints / parameters

Goodness-of-fit on $\mathrm{F}^{2}$

Final $\mathrm{R}$ indices [I $>2 \operatorname{sigma}(\mathrm{I})]$

$\mathrm{R}$ indices (all data)

Extinction coefficient

Largest diff. peak and hole
3328

$0.181 \times 0.148 \times 0.036 \mathrm{~mm}^{3}$

1.174 to $27.500^{\circ}$.

$-28 \leq \mathrm{h} \leq 29,-14 \leq \mathrm{k} \leq 14,-31 \leq \mathrm{l} \leq 31$

85638

$14194[\mathrm{R}(\mathrm{int})=0.0514]$

$100.0 \%$

Integration

0.9073 and 0.6233

Full-matrix least-squares on $\mathrm{F}^{2}$

14194 / 122 / 808

1.050

$\mathrm{R} 1=0.0395, \mathrm{wR} 2=0.0958[11063]$

$\mathrm{R} 1=0.0556, \mathrm{wR} 2=0.1016$

$\mathrm{n} / \mathrm{a}$

2.983 and -1.753 e. $\AA^{-3}$

$R 1=\sum\left(|| F_{0}|-| F_{c}||\right) / \sum\left|F_{0}\right| \quad w R 2=\left[\sum\left[w\left(F_{o}{ }^{2}-F_{c}{ }^{2}\right)^{2}\right] / \sum\left[w\left(F_{o}{ }^{2}\right)^{2}\right]\right]^{1 / 2}$

$S=\left[\Sigma\left[w\left(F_{o}{ }^{2}-F_{c}{ }^{2}\right)^{2}\right] /(n-p)\right]^{1 / 2} \quad w=1 /\left[\sigma^{2}\left(F_{o}{ }^{2}\right)+\left(m^{*} p\right)^{2}+n^{*} p\right], p=\left[\max \left(F_{o}{ }^{2}, 0\right)+2^{*} F_{c}{ }^{2}\right] / 3, m \& n$ are constants. 


\section{Computational Methods}

\section{General Consideration}

All ground state density functional theory (DFT) calculations were performed using the Gaussian 09 program. ${ }^{10}$ The atomic coordinates from the crystal structure of $\mathbf{3}$ served as the input for $\mathbf{3}^{\prime}$. Geometry optimizations of $\mathbf{3}$ were performed in the gas phase employing either (a) the hybrid functional B3LYP ${ }^{11,12}$ along with the triple zeta $\mathrm{TZVP}^{13}$ basis set for all lighter atoms and the $\mathrm{SDD}^{14}$ basis set with pseudopotential for the tungsten atom, or (b) the $\mathrm{M}^{14} 6^{15}$ functional in conjunction with $\mathrm{TZVP}^{13}$ (for lighter atoms) and $\mathrm{SDD}^{14}$ (for tungsten; with pseudopotential) basis sets. Frequency analysis was performed using the same level of theory as the optimization to confirm absence of imaginary frequencies. Single point calculations were also performed at the same level of theory as the optimization to compute orbital images. Molecular orbitals were generated using Gabedit ${ }^{16}$ at the specified isovalues (0.05).

Geometry optimizations of the anionic alkylidyne $\mathbf{4}^{\prime}$ were performed in the gas phase using the input parameters from the geometry optimized structure of $\mathbf{3}^{\prime}$ employing the hybrid functional B3LYP ${ }^{11,12}$ in conjunction with two different basis sets: (i) the triple zeta Def2TZVP ${ }^{13,17}$ basis set for all atoms $(\mathrm{C}, \mathrm{H}, \mathrm{N}, \mathrm{O}, \mathrm{F}$, and $\mathrm{W})$ with pseudopotentials on the $\mathrm{W}$-atom, and (ii) a combination of the $6-31+\mathrm{G}(\mathrm{d}, \mathrm{p})^{18}$ basis set on all lighter atoms $(\mathrm{C}, \mathrm{H}, \mathrm{N}, \mathrm{O}$, and $\mathrm{F})$ the LANL2DZ ${ }^{18-20}$ basis set with pseudopotential for the tungsten atom. Frequency analysis was performed using the same level of theory as the optimization to confirm absence of imaginary frequencies. Single point calculations were also performed at the same level of theory as the optimization to compute orbital images. Molecular orbitals were generated using Gabedit ${ }^{16}$ at the specified isovalues (number of points used during orbital image generation: 200). 
Geometry optimization of the anionic alkylidyne featuring the flexible $\left[\mathrm{CF}_{3}-\mathrm{ONO}\right]^{3-}$ ligand (7') was also performed in the gas phase using the hybrid functional B3LYP in conjunction with the triple zeta Def2TZVP ${ }^{13,17}$ basis set for all atoms $(\mathrm{C}, \mathrm{H}, \mathrm{N}, \mathrm{O}, \mathrm{F}$, and W) with pseudopotentials on the W-atom. Frequency analysis was performed using the same level of theory as the optimization to confirm absence of imaginary frequencies. Single point calculations were also performed at the same level of theory as the optimization to compute orbital images. Molecular orbitals were generated using Gabedit at the specified isovalues (number of points used during orbital image generation: 200).

The optimized structures $\mathbf{4}^{\prime}$ and $\mathbf{7}^{\prime}$ were rationalized through comparison with the previously reported single crystal X-ray structures of anionic tungsten alkylidyne complexes featuring a $[\mathrm{pyr}-\mathrm{ONO}]^{3-}$ ligand $(\mathbf{9}),{ }^{21}\left[\mathrm{O}^{\mathrm{CH} 2} \mathrm{~N}^{\mathrm{CH} 2} \mathrm{O}\right]^{3-} \operatorname{ligand}^{22}(\mathbf{1 0})$, and $\left[\mathrm{ON}^{\mathrm{CH} 2} \mathrm{O}\right]^{3-}$ ligand (11). ${ }^{23}$

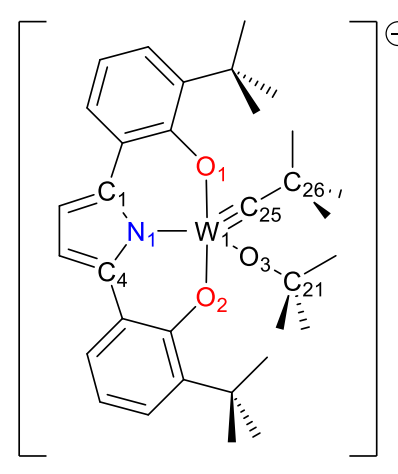

9
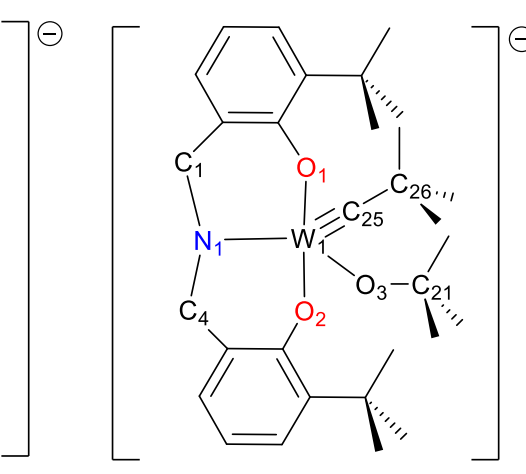

10

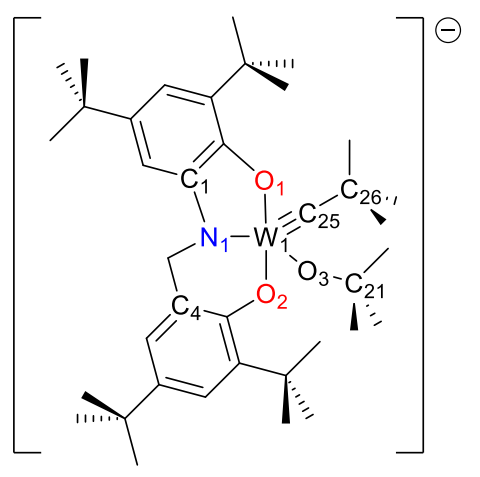

11

Figure S37. Anionic tungsten alkylidyne complexes featuring [pyr-ONO] $]^{3-}$ ligand (9), $\left[\mathrm{O}^{\mathrm{CH} 2} \mathrm{~N}^{\mathrm{CH} 2} \mathrm{O}\right]^{3-}$ ligand $(\mathbf{1 0})$ and $\left[\mathrm{ON}^{\mathrm{CH} 2} \mathrm{O}\right]^{3-}$ ligand (11). 
Table S2. Selected bond lengths $(\AA)$ and angles $\left(^{\circ}\right)$ for $\mathbf{4}^{\prime}, \mathbf{7}^{\prime}$ and single-crystal X-ray structures of $9,10,11$.

\begin{tabular}{|c|c|c|c|c|c|}
\hline Bond length & $\begin{array}{c}\mathbf{4}^{\prime}(\AA) \\
\text { (B3LYP / } \\
\text { Def2TZVP) }\end{array}$ & $\begin{array}{c}7^{\prime}(\AA) \\
\text { (B3LYP / } \\
\text { Def2TZVP) }\end{array}$ & $9(\AA)$ & $10(\AA)$ & $11(\AA)$ \\
\hline $\mathrm{W} 1 \equiv \mathrm{C} 25$ & 1.759 & 1.765 & $1.741(3)$ & $1.758(4)$ & $1.757(3)$ \\
\hline $\mathrm{C} 25-\mathrm{C} 26$ & 1.490 & 1.493 & $1.506(5)$ & $1.515(6)$ & $1.513(4)$ \\
\hline W1-O2 & 1.980 & 1.982 & $1.963(2)$ & $2.011(3)$ & $1.977(18)$ \\
\hline W1-O1 & 1.983 & 1.989 & $1.948(2)$ & $2.018(3)$ & $1.992(18)$ \\
\hline W1-O3 & 1.950 & 1.921 & $1.891(2)$ & $1.934(2)$ & $1.916(18)$ \\
\hline W1-N1 & 2.165 & 2.161 & $2.161(3)$ & $2.026(3)$ & $2.085(2)$ \\
\hline Bond angle & $\begin{array}{l}4^{\prime} \text { (B3LYP / } \\
\text { Def2TZVP) }\end{array}$ & $\begin{array}{l}7^{\prime} \text { (B3LYP / } \\
\text { Def2TZVP) }\end{array}$ & 9 & 10 & 11 \\
\hline$\angle \mathrm{W} 1 \equiv \mathrm{C} 25-\mathrm{C} 26$ & 177.7 & 176.6 & $173.5(3)$ & $173.0(3)$ & $159.7(2)$ \\
\hline$\angle \mathrm{W} 1-\mathrm{O} 3-\mathrm{C} 21$ & 133.3 & 146.9 & $148.9(2)$ & $139.3(3)$ & $139.3(16)$ \\
\hline$\angle \mathrm{N} 1-\mathrm{W} 1-\mathrm{O} 3$ & 159.5 & 149.7 & $152.3(10)$ & $151.3(13)$ & $144.4(8)$ \\
\hline$\angle \mathrm{O} 1-\mathrm{W} 1-\mathrm{O} 2$ & 140.5 & 145.1 & 147.1(9) & $146.5(11)$ & $150.1(8)$ \\
\hline$\angle \mathrm{N} 1-\mathrm{W} 1 \equiv \mathrm{C} 25$ & 98.60 & 101.0 & $96.26(13)$ & $101.7(16)$ & $95.96(11)$ \\
\hline
\end{tabular}

\section{Geometry Optimized Structure of 3'.}

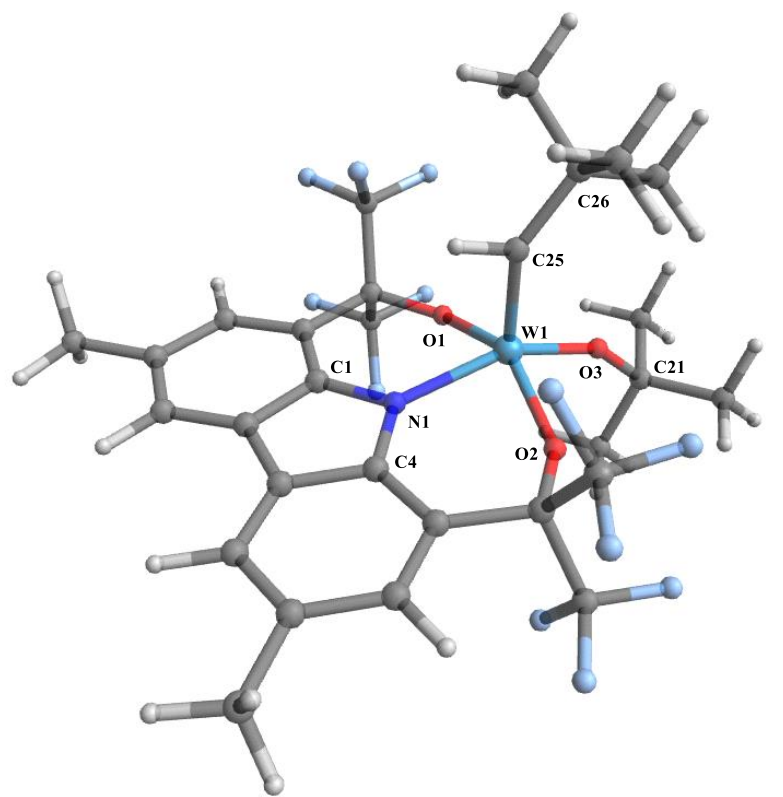

Figure S38. Geometry optimized structure of 3' using B3LYP / triple zeta TZVP / SDD level theory 


\section{Geometry Optimized Structure of 4'.}

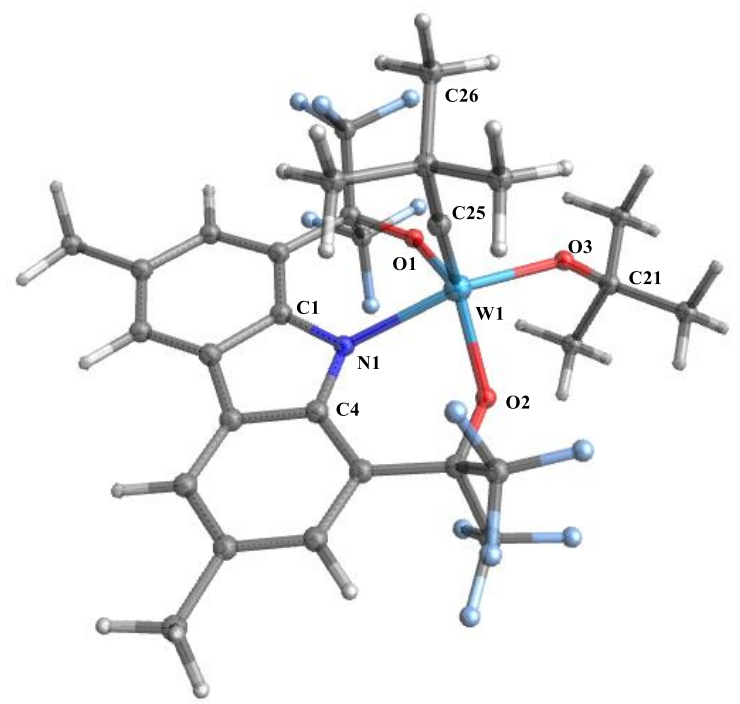

Figure S39. Geometry optimized structure of 4' using B3LYP / triple zeta Def2TZVP level theory.

\section{Geometry Optimized Structure of 7'.}

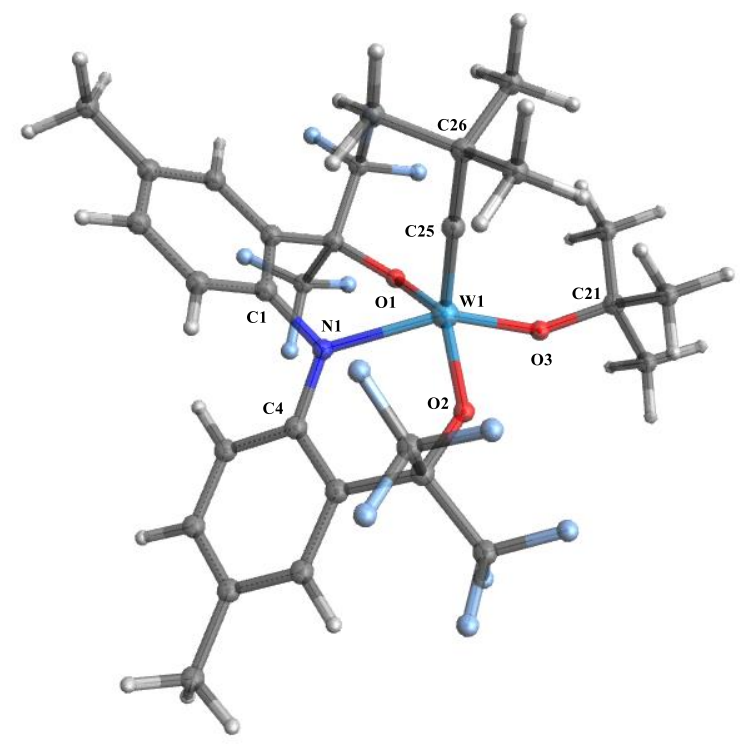

Figure S40. Geometry optimized structure of 7' using B3LYP / triple zeta Def2TZVP level theory. 


\section{References}

1. Britovsek, G. J. P.; Gibson, V. C.; Hoarau, O. D.; Spitzmesser, S. K.; White, A. J. P.; Williams, D. J. Iron and cobalt ethylene polymerization catalysts: Variations on the central donor. Inorg. Chem. 2003, 42, 3454-3465. 10.1021/ic034040q

2. Listemann, M. L.; Schrock, R. R. Multiple metal-carbom bonds. 35. A general-route to tri-tertbutoxytungsten alkylidyne complexes - scission of acetylenes by dutungsten hexa-tert-butoxide. Organometallics 1985, 4, 74-83. 10.1021/om00120a014

3. Bestmann, H. J.; Stransky, W.; Vostrowsky, O. Reactions of alkylidenetriphenylphosphoranes, XXXIII. Preparation of lithium salt free ylide solutions with sodium bis(trimethylsilyl)amide as base. Chem. Ber. Recl. 1976, 109, 1694-1700. 10.1002/cber.19761090513

4. Tonzetich, Z. J.; Schrock, R. R.; Muller, P. Reaction of phosphoranes with Mo(N-2,6-i$\left.\mathrm{Pr}_{2} \mathrm{C}_{6} \mathrm{H}_{3}\right)\left(\mathrm{CHCMe}_{3}\right)\left(\mathrm{OCMe}\left(\mathrm{CF}_{3}\right)_{2}\right)_{2}$ : Synthesis and reactivity of an anionic imido alkylidyne complex. Organometallics 2006, 25, 4301-4306. 10.1021/om060501e

5. O'Reilly, M. E.; Ghiviriga, I.; Abboud, K. A.; Veige, A. S. Unusually stable tungstenacyclobutadienes featuring an ONO trianionic pincer-type ligand. Dalton Transactions 2013, 42, 3326-3336. $10.1039 / \mathrm{c} 2 \mathrm{dt} 32653 \mathrm{a}$

6. O'Reilly, M. E.; Ghiviriga, I.; Abboud, K. A.; Veige, A. S. A new ONO3-trianionic pincer-type ligand for generating highly nucleophilic metal-carbon multiple bonds. J. Am. Chem. Soc. 2012, 134, 1118511195. 10.1021/ja302222s

7. SAINT. Brucker-AXS: Madison, Wisconsin, USA: 2012.

8. Sheldrick, G. M. A short history of SHELX. Acta Crystallograph. A 2008, 64, 112-122. $10.1107 / \mathrm{s} 0108767307043930$

9. Sheldrick, G. M. SHELXT - integrated space-group and crystal-structure determination. Acta Crystallograph. A 2015, 71, 3-8. 10.1107/s2053273314026370

10. Frisch, M. J.; Trucks, G. W.; Schlegel, H. B.; Scuseria, G. E.; Robb, M. A.; Cheeseman, J. R.; Scalmani, G.; Barone, V.; Petersson, G. A.; Nakatsuji, H.; Li, X.; Caricato, M.; Marenich, A.; Bloino, J.; Janesko, B. G.; Gomperts, R.; Mennucci, B.; Hratchian, H. P.; Ortiz, J. V.; Izmaylov, A. F.; Sonnenberg, J. L.; WilliamsYoung, D.; Ding, F.; Lipparini, F.; Egidi, F.; Goings, J.; Peng, B.; Petrone, A.; Henderson, T.; Ranasinghe, D.; Zakrzewski, V. G.; Gao, J.; Rega, N.; Zheng, G.; Liang, W.; Hada, M.; Ehara, M.; Toyota, K.; Fukuda, R.; Hasegawa, J.; Ishida, M.; Nakajima, T.; Honda, Y.; Kitao, O.; Nakai, H.; Vreven, T.; Throssell, K.; Montgomery, J. A.; Peralta, J. J. E.; Ogliaro, F.; Bearpark, M.; Heyd, J. J.; Brothers, E.; Kudin, K. N.; Staroverov, V. N.; Keith, T.; Kobayashi, R.; Normand, J.; Raghavachari, K.; Rendell, A.; Burant, J. C.; Iyengar, S. S.; Tomasi, J.; Cossi, M.; Millam, J. M.; Klene, M.; Adamo, C.; Cammi, R.; Ochterski, J. W.; Martin, R. L.; Morokuma, K.; Farkas, O.; Foresman, J. B.; Fox, D. J. Gaussian 09, Revision D.01. Gaussian, Inc., Wallingford CT: 2016.

11. Becke, A. D. Density-functional thermochemistry. III. The role of exact exchange. J. Chem. Phys. 1993, 98, 5648-5652. 10.1063/1.464913

12. Lee, C. T.; Yang, W. T.; Parr, R. G. Development of the colle-salvetti correlation-energy formula into a functional of the electron-density. Phys. Rev. B. 1988, 37, 785-789. 10.1103/PhysRevB.37.785

13. Weigend, F.; Ahlrichs, R. Balanced basis sets of split valence, triple zeta valence and quadruple zeta valence quality for $\mathrm{H}$ to Rn: Design and assessment of accuracy. Phys. Chem. Chem. Phys. 2005, 7, 32973305. 3297-3305. 10.1039/b508541a

14. Dunning, J. T. H.; Hay, P. J. in: Modern Theoretical Chemistry, Plenum, New York: 1976; pp 1-28. 15. Zhao, Y.; Truhlar, D. G. The M06 suite of density functionals for main group thermochemistry, thermochemical kinetics, noncovalent interactions, excited states, and transition elements: two new functionals and systematic testing of four M06-class functionals and 12 other functionals. Theor. Chem. Acc. 2008, 120, 215-241. 10.1007/s00214-007-0310-x

16. Allouche, A. R. Gabedit-a graphical user interface for computational chemistry softwares. J. Comput. Chem. 2011, 32, 174-182. 10.1002/jcc. 21600 
17. Weigend, F. Accurate coulomb-fitting basis sets for H to Rn. Phys. Chem. Chem. Phys. 2006, 8, 10571065. 10.1039/b515623h

18. Hay, P. J.; Wadt, W. R. Abinitio effective core potentials for molecular calculations - potentials for K to Au including the outermost core orbitals. J. Chem. Phys. 1985, 82, 299-310. 10.1063/1.448975

19. Hay, P. J.; Wadt, W. R. Abinitio effective core potentials for molecular calculations - potentials for the transition-metal atoms Sc to Hg. J. Chem. Phys. 1985, 82, 270-283. 10.1063/1.448799

20. Wadt, W. R.; Hay, P. J. Abinitio effective core potentials for molecular calculations - potentials for main group elements Na to Bi. J. Chem. Phys. 1985, 82, 284-298. 10.1063/1.448800

21. O'Reilly, M. E.; Nadif, S. S.; Ghiviriga, I.; Abboud, K. A.; Veige, A. S. Synthesis and characterization of tungsten alkylidene and alkylidyne complexes supported by a new pyrrolide-centered trianionic $\mathrm{ONO}^{3-}$ pincer-type ligand. Organometallics 2014, 33, 836-839. 10.1021/om4009422

22. VenkatRamani, S.; Huff, N. B.; Jan, M. T.; Ghiviriga, I.; Abboud, K. A.; Veige, A. S. New alkylidyne complexes featuring a flexible trianionic $\mathrm{ONO}^{3-}$ pincer-type ligand: Inorganic enamine effect versus sterics in electrophilic additions. Organometallics 2015, 34, 2841-2848. 10.1021/acs.organomet.5b00155

23. VenkatRamani, S.; Ghiviriga, I.; Abboud, K. A.; Veige, A. S. A new $\mathrm{ONO}^{3-}$ trianionic pincer ligand with intermediate flexibility and its tungsten alkylidene and alkylidyne complexes. Dalton Trans. 2015, 44, 18475-18486. 10.1039/c5dt02911b 